\title{
A new paradigm for intensity modification of tropical cyclones: thermodynamic impact of vertical wind shear on the inflow layer
}

\author{
M. Riemer ${ }^{1}$, M. T. Montgomery ${ }^{1,2}$, and M. E. Nicholls ${ }^{3}$ \\ ${ }^{1}$ Department of Meteorology, Naval Postgraduate School, Monterey, CA, USA \\ ${ }^{2}$ NOAA's Hurricane Research Division, Miami, FL, USA \\ ${ }^{3}$ University of Colorado, Cooperative Institute for Research in Environmental Sciences, Boulder, CO, USA
}

Received: 16 March 2009 - Published in Atmos. Chem. Phys. Discuss.: 4 May 2009

Revised: 2 October 2009 - Accepted: 2 March 2010 - Published: 1 April 2010

\begin{abstract}
An important roadblock to improved intensity forecasts for tropical cyclones (TCs) is our incomplete understanding of the interaction of a TC with the environmental flow. In this paper we re-visit the canonical problem of a TC in vertical wind shear on an f-plane. A suite of numerical experiments is performed with intense TCs in moderate to strong vertical shear. We employ a set of simplified model physics - a simple bulk aerodynamic boundary layer scheme and "warm rain" microphysics - to foster better understanding of the dynamics and thermodynamics that govern the modification of TC intensity. In all experiments the $\mathrm{TC}$ is resilient to shear but significant differences in the intensity evolution occur.

The ventilation of the TC core with dry environmental air at mid-levels and the dilution of the upper-level warm core are two prevailing hypotheses for the adverse effect of vertical shear on storm intensity. Here we propose an alternative and arguably more effective mechanism how cooler and drier (lower $\theta_{e}$ ) air - "anti-fuel" for the TC power machine - can enter the core region of the TC. Strong and persistent, shear-induced downdrafts flux low $\theta_{e}$ air into the boundary layer from above, significantly depressing the $\theta_{e}$ values in the storm's inflow layer. Air with lower $\theta_{e}$ values enters the eyewall updrafts, considerably reducing eyewall $\theta_{e}$ values in the azimuthal mean. When viewed from the perspective of an idealised Carnot-cycle heat engine a decrease of storm intensity can thus be expected. Although the Carnot cycle model is - if at all - only valid for stationary and axisymmetric TCs, a close association of the downward transport of low $\theta_{e}$ into the boundary layer and the intensity evolution offers further evidence in support of our hypothesis.
\end{abstract}

Correspondence to: M. Riemer (mriemer@nps.edu)
The downdrafts that flush the boundary layer with low $\theta_{e}$ air are tied to a quasi-stationary, azimuthal wave number 1 convective asymmetry outside of the eyewall. This convective asymmetry and the associated downdraft pattern extends outwards to approximately $150 \mathrm{~km}$. Downdrafts occur on the vortex scale and form when precipitation falls out from sloping updrafts and evaporates in the unsaturated air below. It is argued that, to zero order, the formation of the convective asymmetry is forced by frictional convergence associated with the azimuthal wave number 1 vortex Rossby wave structure of the outer-vortex tilt. This work points to an important connection between the thermodynamic impact in the near-core boundary layer and the asymmetric balanced dynamics governing the TC vortex evolution.

\section{Introduction}

For decades it has been well known that vertical shear of the environmental wind has a detrimental effect on the intensity of tropical cyclones (TCs). The processes that modulate the intensity of a TC when affected by shear, however, are not well understood. This lack of understanding is partially reflected in high uncertainties that still exist in operational TC intensity forecasts. In addition, recent projections of global climate models indicate an increase in vertical wind shear in the tropical North Atlantic (Vecchi and Soden, 2007). Understanding the interaction of TCs and the vertically sheared environmental flow should thus provide not only a significant benefit to the operational forecast community but may also advance our understanding of changes in TC climate characteristics in a globally warming atmosphere.

Numerous previous studies have been devoted to understand the mechanisms by which a TC-like vortex is resilient to vertical shear. For small amplitude vertical shear forcing

Published by Copernicus Publications on behalf of the European Geosciences Union. 
the differential advection of the vertical shear causes the vortex to tilt and excites vortex Rossby waves (VRWs). Two types of VRW structures have been identified that support the resilience of a TC-like vortex. Propagation of a quasi mode causes the vortex to precess. The exponential decay of this mode leads to vortex alignment. Dispersion and subsequent inviscid damping of sheared VRWs can also lead to the rapid reduction of vortex tilt (Schecter and Montgomery, 2003; Reasor et al., 2004). Moist processes have been suggested to significantly enhance the aforementioned adiabatic resiliency mechanisms (Schecter and Montgomery, 2007). The resiliency of TC-like vortices has also been studied in terms of the interaction of lower and upper-level potential vorticity (PV) anomalies (e.g. Jones, 1995; Smith et al., 2000) and the impact of diabatic processes on these anomalies (Davis et al., 2008).

While these resiliency studies did not focus on the intensity change of a resilient vortex, it was noted that the excitation of the tilt mode weakens the azimuthal mean circulation of the vortex. During the alignment of the vortex, i.e. the decay of the tilt mode, much of the kinetic energy feeds back into the mean circulation. These changes in the strength of the mean vortex, however, are small (e.g. Reasor and Montgomery, 2001). DeMaria (1996) followed the same idea using PV superposition arguments. Using a dry 2-layer model of the troposphere he found an intensity decrease of about $10 \%$ for a very large vortex tilt $(\mathcal{O}(100 \mathrm{~km}))$. In contrast, Wong and Chan (2004) found, in an idealised numerical experiment with a representation of moist processes, a significant intensity decrease with very small tilt. These results suggest that the kinematic effect is not the primary intensity change mechanism.

Wu and Braun (2004) suggested that persistent asymmetries in the TC core that occur due to the interaction with environmental flow weaken the mean circulation by eddy momentum fluxes. Their result was derived from non-cloudresolving numerical experiments with horizontal grid spacing of $25 \mathrm{~km}$ and only considered the kinematic impact of the asymmetries. Very recent work, however, indicates that asymmetries are essential in the intensification process of a TC. Nguyen et al. (2008), performing higher-resolution experiments, identified vortical hot towers (VHTs) as the coherent asymmetric structures in an intensifying TC. In fact, the VHTs carry the local buoyancy that is essential for intensification; while in the azimuthal mean the TC vortex is negatively buoyant relative to its environment (Montgomery et al., 2009). By axisymmetric reasoning, the TC would therefore be expected to weaken, however, it intensifies rapidly in the numerical experiment. We are thus not convinced that the study by $\mathrm{Wu}$ and Braun sufficiently accounts for the role of asymmetries during intensity modification of a TC in vertical shear.

DeMaria (1996) showed, following balance considerations, that a tilted vortex is associated with a warm anomaly at mid-levels leading to a more stable stratification and ar- guably weaker convection. A reduction of the convective vertical mass flux can be expected to lead to a partial spin down of the lower part of the vortex. Jones (2000), however, demonstrated that the temperature anomalies associated with vortex tilt also decrease stability locally and thus might enhance convection. It is not clear that the stability anomaly leads to a decrease of convection in the azimuthal average. In fact, Davis et al. (2008) have shown in 6 simulations of North Atlantic storms that the vertical mass flux increases with shear magnitude up to shear values ${ }^{1}$ of around $15 \mathrm{~ms}^{-1}$.

Emanuel $(1986,1991)$ proposed a theory for the maximum intensity of TCs for axisymmetric, steady flow based on thermodynamic arguments. His theory regards the TC as a heat engine, illustrated in Fig. 1a. Air parcels acquire heat from the ocean surface along the inflow leg (B). The process is supposed to be isothermal. Then, the air parcels are supposed to rise moist-adiabatically in the eyewall and flare outward at upper-levels (C). At large radii it is assumed that air parcels lose enough heat through radiative cooling to return to their ambient $\theta_{e}$ value and that angular momentum is restored (D). The cycle is closed along an absolute vortex line (A) along which the thermodynamic contribution is assumed to be small (Emanuel, 1991, page 184).

The work done by the heat engine (Fig. 2a) is assumed to be converted into kinetic energy that is finally dissipated in the frictional boundary layer (BL). Thus the thermodynamic cycle is linked to the wind field in the BL. A proxy for storm intensity may therefore be derived. Critiques have been raised recently about aspects of the thermodynamic reasoning (Makarieva et al., 2010) and the underlying dynamical treatment of the BL in this theory (Smith et al., 2008). We note further that the closed pathway of air parcels implied in the original Carnot cycle is not realized in our model (neither is it in real storms) and that radiative processes (by which the heat engine is assumed to lose energy at upper-levels) are not represented in our simplified model. We do not intend to use Emanuel's theory in a quantitative manner, but rather only apply its very fundamental idea: that a frustration of the energy cycle yields a decrease in storm intensity.

The development of asymmetries has often been invoked to explain observed decrease in intensity. Much attention is given to asymmetries of vertical motion and precipitation in the eyewall. Earlier studies found a distinct wave number 1 pattern (e.g. Bender, 1997; Frank and Ritchie, 2001; Rogers et al., 2003) while recent work by Braun et al. (2006) documents the internal structure of vertical motion within the wave number 1 envelope. It is, however, not clear why the TC heat engine should not work near peak efficiency in the presence of these asymmetries.

The ventilation of the TC core by dry environmental air at mid-levels was perhaps the earliest idea how TC intensity

\footnotetext{
${ }^{1}$ It is the convention in the TC community to define vertical wind shear as the vector difference between the winds at $200 \mathrm{hPa}$ and $850 \mathrm{hPa}$. The shear magnitude is thus given in $\mathrm{ms}^{-1}$.
} 


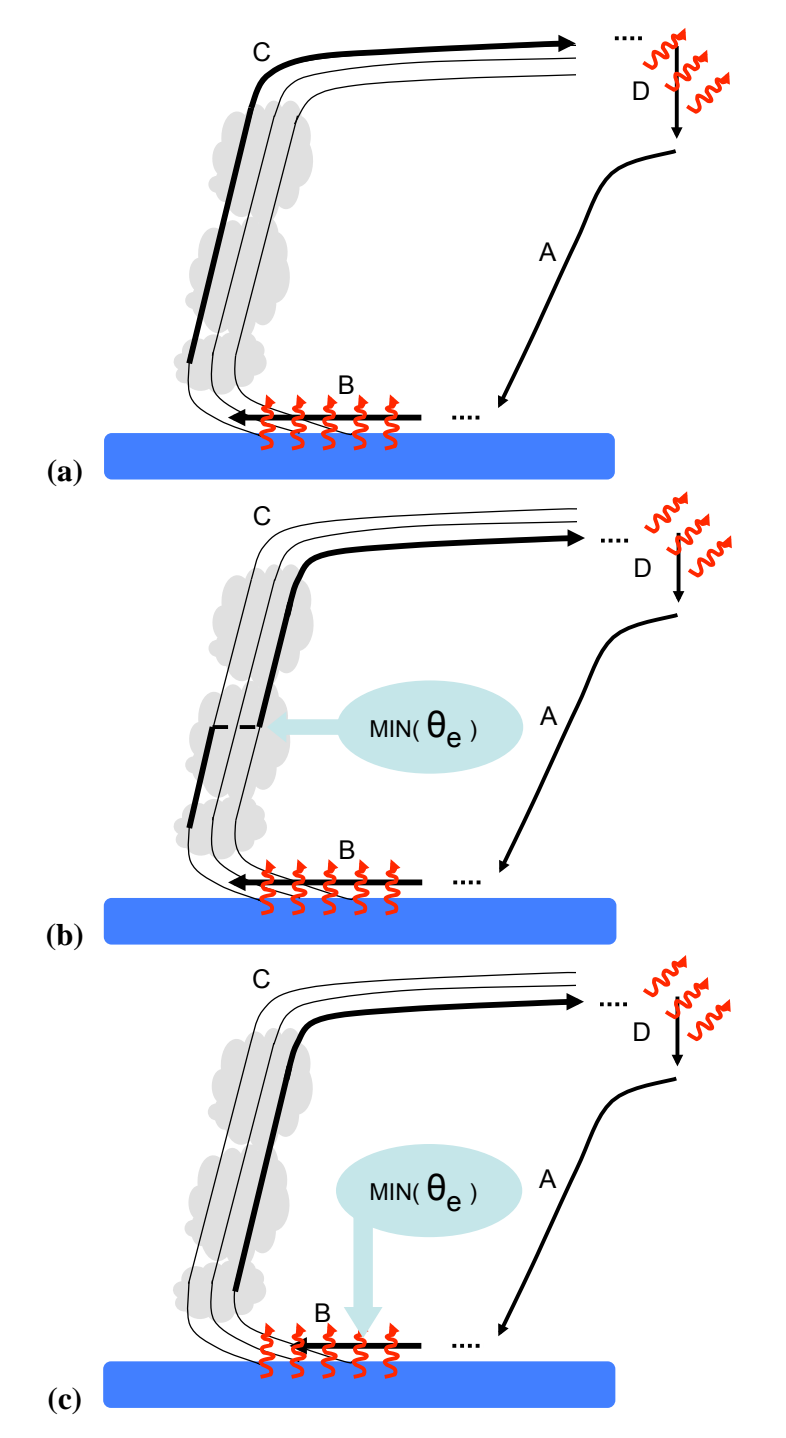

Fig. 1. Schematic in radius-height coordinates of an axisymmetric, steady-state TC working as a Carnot cycle heat engine (Emanuel, 1986) and two hypothesised impacts of vertical shear on the thermodynamic cycle. The storm centre is to the left in each figure and the blue region indicates the ocean. Thick black arrows denote the hypothesised path of air parcels and contours depict isentropes of $\theta_{e}$, decreasing outward from the storm centre. Curved red arrows indicate processes that change the moist entropy (i.e. $\theta_{e}$ ) of an air parcel. (a) The undiluted Carnot cycle. (b) Illustration of the effect of "mid-level ventilation" on the Carnot cycle. Low $\theta_{e}$ air from the $\theta_{e}$ minimum, usually found at mid-levels in the TC environment, is assumed to be transported to and mixed with the air rising in the eyewall on a direct pathway, thereby diluting eyewall $\theta_{e}$ values. Above the level of mixing, air parcels rise on a lower isentrope. (c) Illustration of the pathway proposed in this paper: Downdrafts flush the near-core $\mathrm{BL}$ with low $\theta_{e}$ air originating from above the $\mathrm{BL}$ and thus quench the energy source of the storm in the inflow layer. Surface fluxes do not fully recover $\mathrm{BL} \theta_{e}$ values and air parcels start rising at the base of the eyewall with smaller $\theta_{e}$ values. Both processes (b) and (c) can work concurrently.

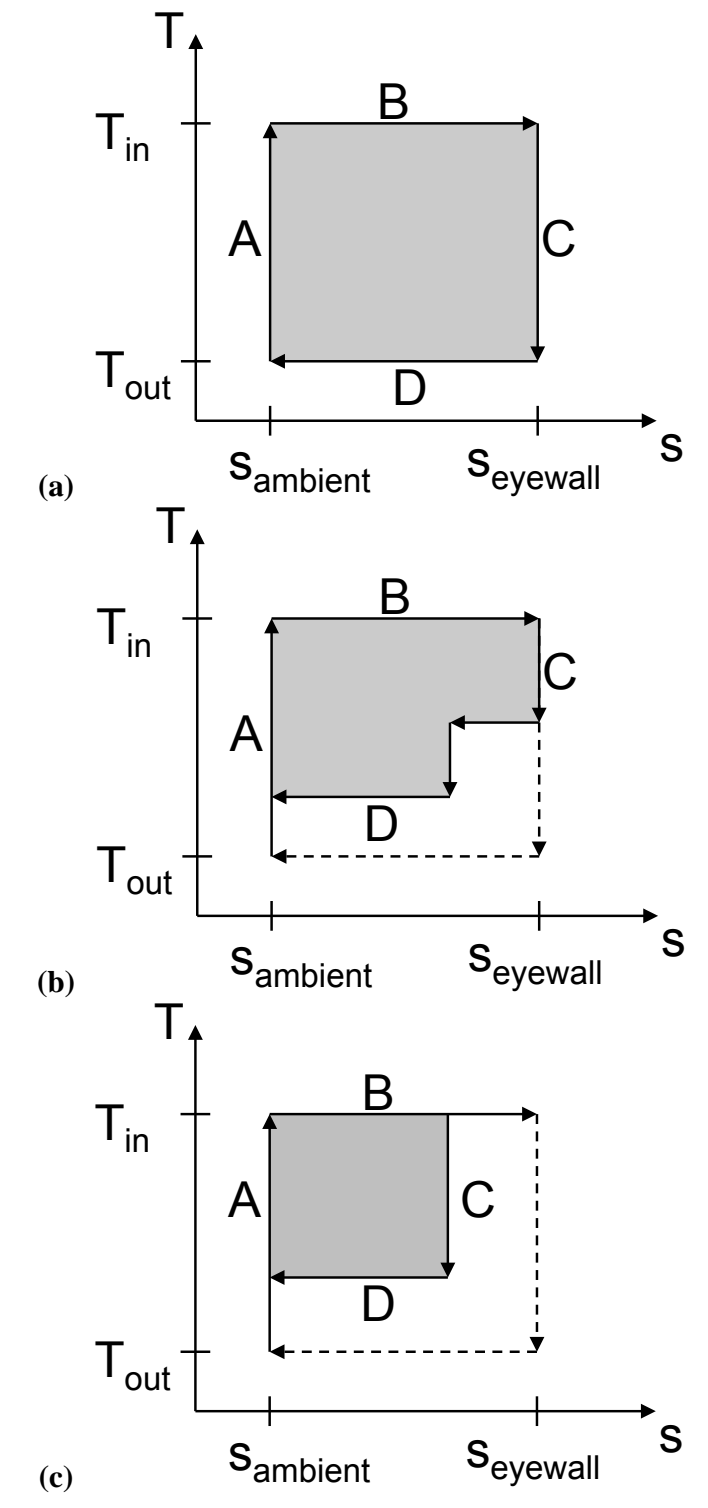

Fig. 2. Schematic temperature $(T)$ - entropy $(s)$ diagrams for the thermodynamic cycles depicted in Fig. $1 . T_{\text {in }}$ and $T_{\text {out }}$ denote the temperature of the inflow and outflow layer, respectively, and $s_{\text {ambient }}$ and $s_{\text {eyewall }}$ denote the moist entropy of ambient air at the level of inflow into the storm and in the eyewall, respectively. The shaded area displays the work that is done by the Carnot cycle and that is assumed to be transformed into kinetic energy. This area thus indicates a proxy for the intensity of the TC that can be expected on the grounds of Emanuel's steady-state intensity theory. (a) The undiluted cycle, (b) effect of mid-level ventilation, and (c) flushing of the near-core $\mathrm{BL}$ with low $\theta_{e}$ air. The diagrams are reproduced from Brian Tang's PhD thesis proposal at MIT with permission. 
could be constrained by vertical shear (Simpson and Riehl, 1958). Mixing of low $\theta_{e}$ air into the eyewall at mid-levels is thought to be particularly effective because the minimum of $\theta_{e}$ is usually located at this level (Fig. 1b). The frustration of eyewall $\theta_{e}$ values would constitute a dilution of the fuel burnt in the TC engine and thus reduce the amount of work produced (Fig. 2b). A somewhat similar hypothesis is the dilution of the upper-level warm core by outward fluxes of high $\theta_{e}$ and PV values and the subsequent weakening of the vortex from the top to bottom, invoking hydrostatic arguments (Frank and Ritchie, 2001). Wong and Chan (2004) expect the dilution of the upper-level core to be enhanced when the shear is strong enough to suppress eyewall updrafts on the upshear side. In terms of the TC heat engine, however, the effectiveness of upper-level $\theta_{e}$ mixing is questionable because the radial $\theta_{e}$ gradient is less pronounced. Furthermore, a TC constitutes a strong atmospheric vortex. Air within the vortex tends to be contained and sheltered from intrusion from the environment, in part due to the strong radial shear of the tangential wind. Any asymmetry that tries to invade the vortex tends to be damped by the action of differential shear: This is the so-called vortex axisymmetrisation process (Melander et al., 1987; Carr and Williams, 1989) that is a well known essential ingredient in the robustness and persistence of coherent vortex structures in quasi two-dimensional flows. This aspect has not been explicitly discussed in the studies mentioned above in this paragraph. A trajectory analysis by Cram et al. (2007) in a moderately sheared TC $\left(10-12 \mathrm{~ms}^{-1}\right)$ found a reduction of eyewall $\theta_{e}$ due to environmental entrainment of $\mathcal{O}(1) \mathrm{K}$. This small value illustrates the limitation of air parcel exchange between the environment and the eyewall in this case as mid-level $\theta_{e}$ values of the storm environment are typically more than $15 \mathrm{~K}$ less than that found in the eyewall of mature storms.

Here we re-visit the canonical idealised numerical experiment of a TC in vertical shear (e.g. Bender, 1997; Frank and Ritchie, 2001). In the now standard posing of the problem a TC, after being spun up in quiescent environment, is suddenly exposed to a vertical wind shear profile. We intentionally employ a very simple set of physical parameterisations that, as we argue, capture the essence of the TC - shear interaction and help form the basis for an understanding of the problem. A new hypothesis for intensity modification of a TC interacting with vertical shear is proposed and supporting evidence from our experiments presented. The energy cycle is frustrated at its most vulnerable part: the inflow layer of the TC. Strong and persistent downdrafts bring low $\theta_{e}$ air into the $\mathrm{BL}$ of the storm, significantly depressing near-core $\mathrm{BL} \theta_{e}$ values (Fig. 1c). Latent and sensible heat fluxes from the warm ocean surface do not recover $\theta_{e}$ values completely, as compared to the undiluted inflow. Air parcels in the eyewall start rising with reduced $\theta_{e}$ values (Fig. 2c). The flushing of the BL with low- $\theta_{e}$ air - anti-fuel for the TC's engine - is capable of leading to intensity changes of tens of $\mathrm{ms}^{-1}$ (and $\mathrm{hPa}$ ), without the vortex being sheared apart.
The remainder of this paper is organised as follows. Section 2 describes the numerical model employed, the posing of the problem, and the initial conditions. An overview of the evolution in our experiments is then provided in Sect. 3. The details of the thermodynamic impact on the BL and the structure of the associated downward flux of low $\theta_{e}$ are presented in Sect. 4. Section 5 discusses the formation of the persistent downdrafts outside of the eyewall and shows evidence that the vortex-scale downdraft distribution is guided by balanced vortex dynamics. In Sect. 6, we comment on the near-core shear evolution and the storm's re-intensification. A discussion of the results and some observational support for the new paradigm is given in Sect. 7 before we present our conclusions in Sect. 8.

\section{Experimental setup}

\subsection{Numerical model formulation}

For the numerical experiments we employ the Regional Atmospheric Modeling System (RAMS), developed at Colorado State University (Pielke and Coauthors, 1992; Cotton and Coauthors, 2003). The RAMS is a threedimensional non-hydrostatic numerical modeling system comprising time-dependent equations for velocity, nondimensional pressure perturbation, ice-liquid water potential temperature (Tripoli and Cotton, 1981), and mixing ratios of total water and rain. Cloud water and water vapor mixing ratio, and potential temperature are diagnosed. The model is formulated on an Arakawa C-grid.

We use a simple cloud microphysics scheme that is based on the scheme of Kessler (1969) and considers warm cloud processes only. Rain and cloud water may evaporate in unsaturated air. A standard first-order turbulence scheme developed by Smagorinsky (1963) is used for sub-grid-scale mixing, with modifications by Lilly (1962) and Hill (1974) that enhance diffusion in unstable conditions and reduce diffusion in stable conditions. For simplicity, radiative processes are neglected. First, we believe that they play a minor role only in the evolution of a TC in vertical shear. Further, considerable heat fluxes from the ocean are confined to a relatively small area around the TC, as compared to the size of our computational domain and thus radiative cooling is not needed to balance a negligible warming of the domain during the course of our integration.

The non-hydrostatic RAMS equations are given as follows: The vector momentum Eq. is

$\frac{\partial \boldsymbol{v}}{\partial t}=-\boldsymbol{v} \nabla \boldsymbol{v}-\theta_{v 0} \nabla \Pi^{\prime}+f \boldsymbol{v} \cdot \boldsymbol{k}$
$+g\left[\frac{\theta_{v}^{\prime}}{\theta_{v 0}}-\left(r_{t}-r_{v}\right)\right] \boldsymbol{k}+\nabla\left(K_{m} \nabla \boldsymbol{v}\right)$.

The thermodynamic Eq. is

$\frac{\partial \theta_{i l}}{\partial t}=-\boldsymbol{v} \nabla \theta_{i l}+\nabla\left(K_{h} \nabla \theta_{i l}\right)+\mathcal{S}_{p r}$. 
The water species mixing ratio equations are

$\frac{\partial r_{n}}{\partial t}=-\boldsymbol{v} \nabla r_{n}+\nabla\left(K_{h} \nabla r_{n}\right)+\mathcal{S}_{c}$.

The mass continuity Eq. is

$\frac{\partial \Pi^{\prime}}{\partial t}=-\frac{R \Pi_{0}}{c_{v} \rho_{0} \theta_{v 0}}\left[\nabla\left(\rho_{0} \theta_{v 0} \boldsymbol{v}\right)\right]$.

Here $v$ is the three-dimensional velocity vector, $\theta$ is potential temperature, the Exner function

$\Pi=c_{p}\left(\frac{p}{p_{r}}\right)^{\frac{R}{c_{p}}}$,

with total pressure $p$, the reference pressure $p_{r}=1000 \mathrm{hPa}$, $R$ is the gas constant for dry air, $c_{p}$ the specific heat capacity for dry air at constant pressure, $f$ the Coriolis parameter, $\theta_{v}$ is virtual potential temperature, $r_{t}$ the total water mixing ratio, $r_{v}$ the vapor mixing ratio, $K_{m}$ and $K_{h}$ the eddy viscosity coefficient for momentum and enthalpy, respectively, $\theta_{i l}$ denotes the ice-liquid water potential temperature (as we do not consider the ice phase, $\theta_{i l}$ reduces to liquid water potential temperature, see Eq. 6), $\mathcal{S}_{p r}$ represents a source/sink term due to precipitative flux (see Tripoli and Cotton, 1981), $r_{n}$ mixing ratios of total water $\left(r_{t}\right)$ and rain $\left(r_{r}\right), \mathcal{S}_{c}$ a source/sink term from conversion of water species and precipitation, $c_{v}$ the specific heat capacity for dry air at constant volume and $\rho$ is air density. Thermodynamic variables are decomposed into a horizontal reference state (denoted by subscript zero) from which the predicted deviations (denoted by a prime) should be small. To represent the interaction between the model atmosphere and the underlying sea surface the momentum and thermodynamic equations (Eq. 1 and 2, respectively) are further modified by surface fluxes as discussed below (Sect. 2.3).

The potential temperature is diagnosed as:

$\theta=\theta_{i l}\left[1+\frac{L_{v} r_{l}}{c_{p} \max (T, 253)}\right]$.

The mixing ratio of liquid water $r_{l}=r_{c}+r_{r}$ (where $r_{c}$ is the cloud water mixing ratio) and $L_{v}$ is the latent heat of condensation. Water vapor is diagnosed from

$r_{v}=r_{t}-r_{r}-r_{c}$

and cloud water from

$r_{c}=\max \left(r_{t}-r_{r}-r_{v s a t}, 0\right)$

where the saturation mixing ratio is given by

$r_{v s a t}=\frac{0.622 e_{s v}}{p-e_{s v}}$.

The saturated vapor pressure $e_{s v}$ is a function of temperature $T$ and calculated from the Clausius-Clapeyron Eq. Finally, temperature is diagnosed from
$T=\frac{\theta \Pi}{c_{p}}$.

Equations 6-10 are solved iteratively.

Cloud water is assumed to move with the air while rain is also allowed to sink relative to the air. The terminal velocity increases as a power of the droplet diameter and is based on empirical data. In the absence of rain, cloud water forms as soon as saturation is exceeded (Eq. 8). Clouds form on a prescribed distribution of cloud condensation nuclei. An autoconversion parameterisation that depends on the cloud water mixing ratio and the mean diameter of the cloud droplets is used to represent the formation of rain by collision and coalescence of cloud droplets.

\subsection{Grid configuration}

RAMS utilises the two-way interactive multiple nested grid scheme developed by Clark and Farley (1984). This allows explicit representation of cloud-scale features within the finest grid while enabling a large domain size to be used, thereby minimizing the impact of lateral boundary conditions. A standard radiation boundary condition is used at the lateral boundaries, which assumes that disturbances reaching the boundary move as linearly propagating gravity waves (Klemp and Wilhelmson, 1978). A Rayleigh friction layer is included at upper-levels (above $17 \mathrm{~km}$ ) to minimize the reflection of gravity waves from the top of the model. At the lower boundary the vertical velocity is required to vanish.

\subsection{Surface interaction}

The bulk aerodynamic formulas are used to calculate the zonal $\left(\tau_{u}\right)$ and meridional $\left(\tau_{v}\right)$ momentum and sensible $\left(\tau_{\theta}\right)$ and latent $\left(\tau_{r v}\right)$ heat fluxes from the sea surface.

$$
\begin{aligned}
& \tau_{u}=-C_{D}|V|^{1} u^{1} \\
& \tau_{v}=-C_{D}|V|^{1} v^{1} \\
& \tau_{\theta}=-C_{K}|V|^{1}\left(\theta^{1}-\theta^{s}\right) \\
& \tau_{r v}=-C_{K}|V|^{1}\left(r_{v}^{1}-r_{v s a t}^{s}\right) .
\end{aligned}
$$

The superscripts ${ }^{1}$ and ${ }^{s}$ denote values at the lowest model level and at the surface, respectively, and $|V|$ the horizontal wind speed. The vertical divergences of the fluxes (11-14) enter the momentum, thermodynamic, and the water species mixing ratio equations (Eqs. 1-3), respectively) as tendencies at the lowest model level. The vertical flux divergence is calculated as $\tau / h^{w 1}$, where $h^{w 1}$ denotes the height of the lowest model level of vertical motion ${ }^{2}$ above the ground, located at $100 \mathrm{~m}$.

The drag coefficient is represented here by Deacon's formula:

$C_{D}=1.1 \times 10^{-3}+4 \times 10^{-5}|V|^{1}$.

\footnotetext{
${ }^{2}$ On the Arakawa C-grid, vertical velocity is staggered in the vertical with respect to the remaining model variables.
} 
$C_{K}$ is the exchange coefficient of enthalpy with the ratio

$$
\frac{C_{K}}{C_{D}}=1 .
$$

The latest findings on the dependence of these exchange coefficients on wind speed (Black et al., 2007; Zhang et al., 2008) indicate that, in particular for high wind speeds, $\frac{C_{K}}{C_{D}}<$ 1. These new results should be incorporated in future tests of our findings with respect to uncertainties in the model parameterisations. Here we speculate that a decrease of $C_{K}$ in the higher wind regime would actually emphasise the importance of our proposed mechanism: depressed $\operatorname{BL} \theta_{e}$ values would be replenished more slowly and their effect on the eyewall updrafts, and thus intensity, would be more pronounced.

\subsection{Model domain and initial conditions}

We use 3 domains with a horizontal grid spacing of $45 \mathrm{~km}, 15 \mathrm{~km}$ and $5 \mathrm{~km}$, respectively. The outermost domain contains $120 \times 120$ grid points giving a domain size of $5355 \times 5355 \mathrm{~km}$. The second and third domains have $200 \times 122(2885 \times 1815 \mathrm{~km})$ and $401 \times 161(2000 \times 800 \mathrm{~km})$ grid points, respectively. Both inner grids are centred within the outermost domain. We use 38 vertical levels with the lowest level at $49 \mathrm{~m}$. The vertical resolution is $100 \mathrm{~m}$ between the lowest two levels and decreases with height by a stretching factor of 1.09 between grid levels. The top of the model resides at $24.6 \mathrm{~km}$. The model domain is on an f-plane and the Coriolis parameter valid for $15^{\circ} \mathrm{N}$. A modified Jordan sounding (Jordan, 1958) is used as the background sounding. While the temperature profile is retained the low-level moisture is reduced with a maximum drying of $2 \mathrm{gkg}^{-1}$ at the surface. The low-level drying is employed to retard convective activity not associated with the TC. The time-invariant SST is set to $28.5^{\circ} \mathrm{C}$.

The initial vortex has an axisymmetric structure with a maximum wind speed of $20 \mathrm{~ms}^{-1}$ at radius $135 \mathrm{~km}$ and $4 \mathrm{~km}$ height. The maximum surface wind speed is $18 \mathrm{~ms}^{-1}$ and the vortex has a depth of $10 \mathrm{~km}$. For a radius less than $75 \mathrm{~km}$ the vortex is moistened to $95 \%$ of saturation between the surface and $526 \mathrm{~m}$, and to $90 \%$ of saturation above. Between a radius of 75 to $100 \mathrm{~km}$ the moisture anomaly is linearly reduced to zero. The initial vortex is centred in the fine grid in the meridional direction. In anticipation of a predominantly westward movement in the shear experiments, the vortex is initially placed in the eastern part of the innermost nest, $250 \mathrm{~km}$ away from the transition to grid 2 .

\subsection{Vertical shear flow in thermal wind balance}

For the shear experiments we superimpose a wind field in thermal wind balance after the vortex spin up phase. The shear profile is given by a cosine structure:

$U(z)=U_{m}\left[1-\cos \left(\frac{\pi z}{H}\right)\right]$ for $z \leq H$,
$U(z)=2 U_{m}$ for $z>H$.

The associated temperature and pressure perturbations in thermal wind balance with the shear profile are given by

$$
\begin{aligned}
& \theta_{v}^{\prime}(y, z)=\theta_{v 0} \frac{f y}{g} U_{m} \\
& \times\left[\frac{1}{\theta_{v 0}} \frac{d \theta_{v 0}}{d z}-\frac{\pi}{H} \sin \left(\frac{\pi z}{H}\right)-\cos \left(\frac{\pi z}{H}\right) \frac{1}{\theta_{v 0}} \frac{d \theta_{v 0}}{d z}\right] \\
& \Pi^{\prime}(y, z)=-\frac{f y}{\theta_{v 0}} U_{m}\left[1-\cos \left(\frac{\pi z}{H}\right)\right]
\end{aligned}
$$

below $z=H$ and by

$\theta_{v}^{\prime}=-\frac{f y}{g} 2 U_{m} \frac{d \theta_{v 0}}{d z}$

$\Pi^{\prime}=\frac{f y}{\theta_{v 0}} 2 U_{m}$

above. We use $12 \mathrm{~km}$ as the height $H$ of maximum wind $2 U_{m}$. The velocity change between the surface and $12 \mathrm{~km}$ height is thus given by $2 U_{m}$. For our shear profile this corresponds approximately to the commonly used shear value between $850 \mathrm{hPa}$ to $200 \mathrm{hPa}$. Here we conduct experiments with $2 U_{m}$ values of $0,-10,-15$, and $-20 \mathrm{~ms}^{-1}$; these experiments will hereafter be referred to as the no_shear, $10 \mathrm{mps}, 15 \mathrm{mps}$, and $20 \mathrm{mps}$ case, respectively. The negative values denote easterly shear. Note that this profile exhibits zero surface wind and shear.

A slight imbalance occurs when superposing the balanced field on the tropical cyclone after the spin up phase. We do not see any indication that this imbalance compromises our experiment or subsequent physical interpretations after the short adjustment time to the imposed shear.

We have performed a shear experiment with $2 U_{m}=$ $-5 \mathrm{~ms}^{-1}$ also. None of the key structural changes that occur in the $10 \mathrm{mps}, 15 \mathrm{mps}$, and $20 \mathrm{mps}$ cases (discussed below) are found in this experiment. The intensity evolution in this case appears to be within the ensemble envelope of intensification for a quiescent environment as reported by Nguyen et al. (2008). In our model, the TC is approx. Cat 4 when the shear is imposed and continues to intensify to over $95 \mathrm{~ms}^{-1}$ in the no_shear case (Fig. 3). It is thus reasonable that such an intense storm is hardly affected by moderately weak shear. We hypothesise that the vortex response scales in proportion to the ratio of storm intensity/ shear magnitude, i.e. the basic phenomenology will be qualitatively similar for less intense storms in weaker shear. Ongoing work is dedicated to test this hypothesis. ${ }^{3}$

\footnotetext{
${ }^{3}$ So far, we have performed one experiment in which shear $\left(2 U_{m}=-15 \mathrm{~ms}^{-1}\right)$ is imposed after $30 \mathrm{~h}$ of spin up when the TC is approx. $20 \mathrm{~ms}^{-1}$ less intense than in the $15 \mathrm{mps}$ case. The subsequent decrease in intensity is indeed more pronounced than for the $15 \mathrm{mps}$ case reported in this paper. Further details on this experiment can be found in our response to John Molinari on the ACPD webpage.
} 


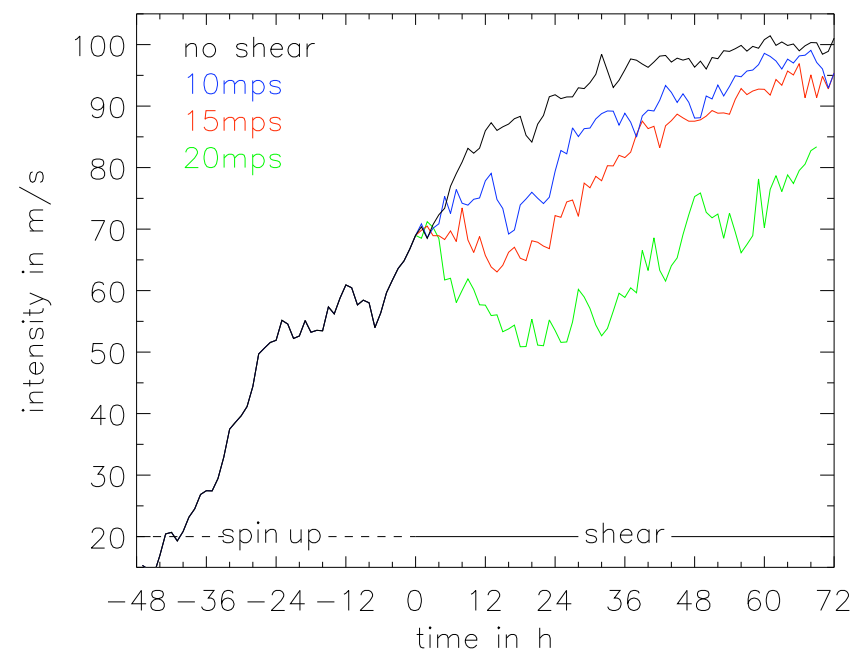

Fig. 3. Intensity time series for the spin up period $(-48 \mathrm{~h}-0 \mathrm{~h})$ and the respective shear experiments. Shear is imposed at $0 \mathrm{~h}$. In this study, intensity is defined as the azimuthal mean tangential wind speed at $1 \mathrm{~km}$ height.

\section{Overview of storm evolution}

In this section we give an overview of the simulated TC evolution in terms of intensity, track, and vortex tilt in the respective experiments.

The intensity measure in this study is the maximum azimuthal average of the tangential wind in $1 \mathrm{~km}$ height. The centre of the storm is calculated iteratively as the centroid of positive relative vorticity in a $120 \mathrm{~km} \times 120 \mathrm{~km}$ box around the centre, averaged over the lowest $2 \mathrm{~km}$. The surface pressure minimum is used as the initial first guess centre of the square. The centre of the square is adjusted and the vorticity centroid recalculated if the centroid differs more than half a grid length from the previous one. Data from the innermost nest is used and the centroid calculation usually converges after $2-3$ steps. The tilt of the vortex is calculated as the difference between the position vectors of the vorticity centroid at $10 \mathrm{~km}$ and $1 \mathrm{~km}$ height.

\subsection{Spin up period and evolution without environmental shear}

Following an initial gestation period of $6 \mathrm{~h}$ during which the BL moistens, the initial vortex intensifies rapidly to $50 \mathrm{~ms}^{-1}$ in the first $20 \mathrm{~h}$ (Fig. 3). During this period the structure of the incipient TC is dominated by VHTs, i.e. distinct and co-located maxima of updrafts, cyclonic vorticity, and $\theta_{e}$, at radii between $60 \mathrm{~km}-80 \mathrm{~km}$ (not shown). At the end of this period these localised vorticity maxima have aggregated to form a ring-like structure with a local minimum of vorticity and $\theta_{e}$ in the centre. The storm evolution in the next $10 \mathrm{~h}$ is dominated by a redistribution of the high values of vorticity

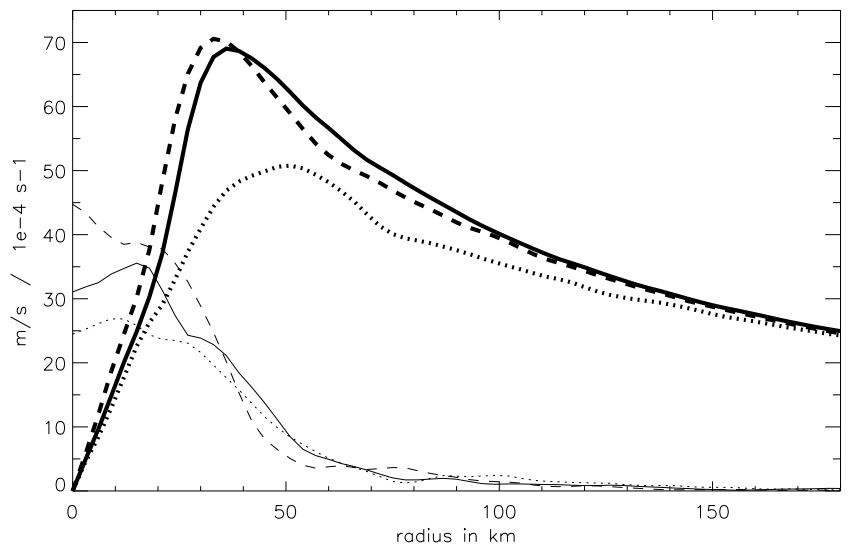

Fig. 4. Radial profiles of azimuthally averaged vorticity above the $\mathrm{BL}$ at $2 \mathrm{~km}$ height (thin) and tangential wind speed at $1 \mathrm{~km}$ height (thick) at time $0 \mathrm{~h}$ (solid), at $2 \mathrm{~h}$ (dashed) and at $18 \mathrm{~h}$ (dotted) for the $20 \mathrm{mps}$ case.

and $\theta_{e}$ from the ring into the centre (similar to Schubert et al., 1999). The resulting incipient vorticity monolith first has an elliptic structure, which then undergoes axisymmetrisation by a pronounced shedding of rain bands, arguably propagating VRWs that are coupled to the BL and deep moist convection (Montgomery and Kallenbach, 1997; Chen and Yau, 2001; Wang, 2002). During this period ( $20 \mathrm{~h}-40 \mathrm{~h}$ ) the storm intensifies at a much slower rate. At $40 \mathrm{~h}$ the structure of the storm has become similar to the one shown in Fig. 7a. A second period of rapid intensification ensues in which the TC intensifies to $80 \mathrm{~ms}^{-1}$ at $60 \mathrm{~h}$. The storm continues to intensify gradually and reaches a quasi-steady intensity of about $95 \mathrm{~ms}^{-1}-100 \mathrm{~ms}^{-1}$ after $84 \mathrm{~h}$.

As in the study by Frank and Ritchie (2001), the environmental wind profile in the shear experiments is superimposed at $48 \mathrm{~h}$. We note that this is during a time of rapid intensification. In the following we only consider the time period after the shear is imposed, i.e. the time after $48 \mathrm{~h}$. We hereafter refer to this time as $0 \mathrm{~h}$ and subsequent times are given in relation to this point in time.

To illustrate the vortex structure at $0 \mathrm{~h}$ the azimuthally averaged radial profiles of tangential wind at $1 \mathrm{~km}$ and vorticity above the BL at $2 \mathrm{~km}$ height are depicted in Fig. 4 (solid lines). The relative vorticity distribution has a bowl-shaped structure in the inner core and the radial gradient of vorticity changes its sign at approx. $20 \mathrm{~km}$ radius. The vorticity profile thus allows for barotropic instability. It has been suggested (e.g. Schubert et al., 1999) that mixing of vorticity and also $\theta_{e}$ in the inner core can lead to TC intensity changes. In the sheared cases simulated here the vorticity profile predominantly shows a monopole structure soon $(2-4 \mathrm{~h})$ after the shear is imposed (thin dashed line in Fig. 4). This is consistent with vorticity mixing in the inner core early in the shear experiments. The intensity modification, however, is minor 


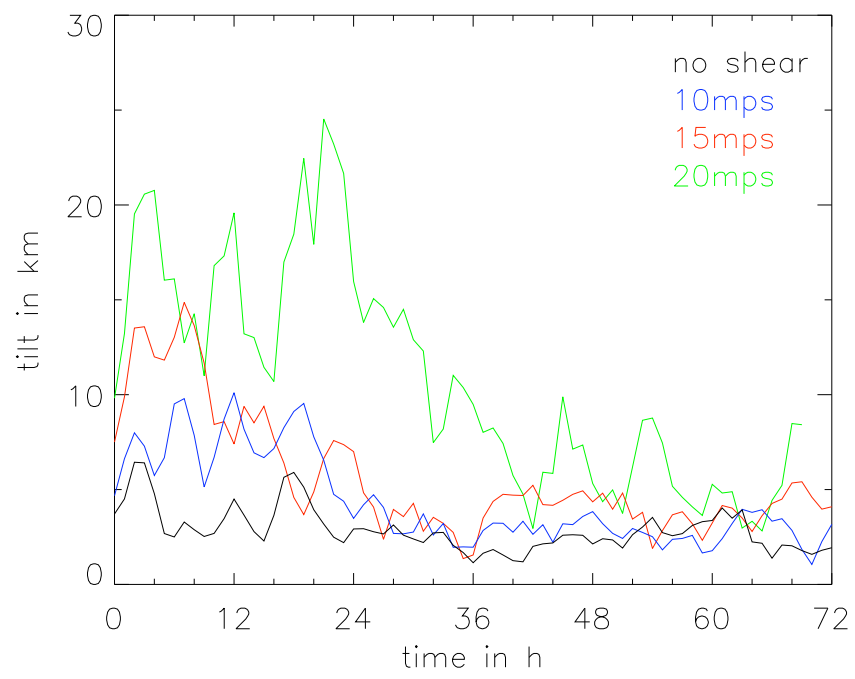

Fig. 5. Magnitude of vortex tilt for the no_shear (black), 10mps (blue), $15 \mathrm{mps}$ (red), and 20mps case (green), respectively. For clarity, the $3 \mathrm{~h}$ running mean of the time series is shown.

during this mixing period and actually the storm slightly intensifies (thick dashed line in Fig. 4). The decrease in intensity associated with vertical shear is found on a much longer time scale of 12-24 h (see below). We are thus confident that vorticity mixing processes in the inner core region are not a major component of the significant intensity modification in our experiments. The vorticity and wind profiles after significant weakening are exemplified in Fig. 4 by the dotted lines (at $18 \mathrm{~h}$ ).

After the spin up period the vortex tilt in the no_shear case is below $5 \mathrm{~km}$ except for two peaks at $3 \mathrm{~h}(10 \mathrm{~km})$ and $18 \mathrm{~h}$ $(8 \mathrm{~km})$ (Fig. 5). The tilt direction indicates a high-frequency internal precession mode ${ }^{4}$ of 3-4h (not shown). These intrinsic tilt characteristics are the benchmark against which the tilt evolution in the sheared cases will be compared.

\subsection{Shear cases}

\subsubsection{Storm movement}

The vertical shear profile constitutes a steering flow for the TC. The TCs move with an average speed of $3.5 \mathrm{~ms}^{-1}$, $5 \mathrm{~ms}^{-1}$, and $6.5 \mathrm{~ms}^{-1}$ in the $10 \mathrm{mps}, 15 \mathrm{mps}$, and $20 \mathrm{mps}$ case, respectively. The storm tracks within the innermost domain are depicted in Fig. 6 . In the 20mps case, the TC centre approaches the western boundary closely and the experiment is stopped at $69 \mathrm{~h}$. The tracks of the storms have a southward component, likely due to a combination of the downshear displacement of the outflow anticyclone (see also Sect. 6) and

\footnotetext{
${ }^{4}$ The precession frequency is estimated from hourly data of the tilt direction. It is possible that the true precession frequency is not resolved due to insufficient temporal sampling
}

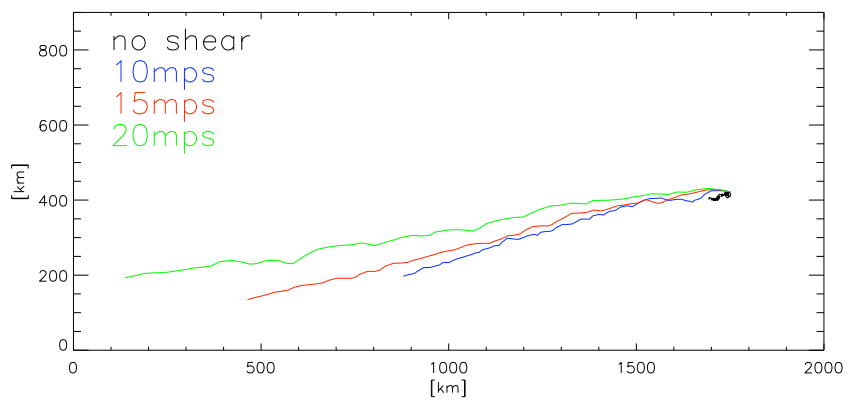

Fig. 6. TC tracks for the $20 \mathrm{mps}$ (green), $15 \mathrm{mps}$ (red), 10mps (blue) and the no_shear case (black) in the innermost model domain for the shear period from $0-72 \mathrm{~h}(69 \mathrm{~h}$ for $20 \mathrm{mps}$ due to proximity to the domain boundary).

the " $\beta$-drift" on the gradient of potential vorticity associated with the environmental shear flow.

\subsubsection{Intensity evolution and storm resiliency}

An imposition of moderate to strong shear hinders the rapid intensification ${ }^{5}$ ( $10 \mathrm{mps}$ case) or leads to an intensity decrease (15mps and 20mps cases, Fig. 3). In all of these cases, the simulated storms re-intensify after a period of weakening. We offer further comments on the recovery of the storms in Sect. 6 . In the $10 \mathrm{mps}$ and $15 \mathrm{mps}$ cases the intensity reaches maximum values only slightly $\left(\approx 5 \mathrm{~ms}^{-1}\right)$ less than in the no_shear case. In the $20 \mathrm{mps}$ case the maximum intensity at the end of the experiment is significantly $\left(20 \mathrm{~ms}^{-1}\right)$ less than in the no_shear case.

Using the azimuthal mean tangential wind speed at $1 \mathrm{~km}$ height as the intensity metric, the TC intensity decreases with higher shear values. A more complex intensity behavior is found in terms of the maximum local wind speed at $1 \mathrm{~km}$ height. Using this latter metric, the most intense storm is found in the $15 \mathrm{mps}$ case: the local wind maximum is $10 \mathrm{~ms}^{-1}$ stronger than in the no_shear case at the end of the experiment (not shown). The location and evolution of the local wind maxima in sheared TCs have, of course, considerable implications at landfall and possibly for the generation of storm surge and are thus important subjects for future research.

Although other studies have found TCs to decay at smaller values of vertical shear (e.g. Frank and Ritchie, 2001), the resiliency of the vortex in the higher shear regime is not a unique feature of our experiments. Wang et al. (2004) found resilient TCs for shear values up to $17 \mathrm{~ms}^{-1}$ in a very similar experimental setup. In a simulation of Hurricane Erin by Braun and Wu (2007) the model TC did not decay in a shear

\footnotetext{
${ }^{5}$ The suspension of intensification constitutes a weakening relative to the no_shear case and will be referred to as weakening in the following.
} 
environment of $20 \mathrm{~ms}^{-1}$ (the authors noted that the shear in their simulation was greater than in reality). In a simulation of Hurricane Bonnie (Braun et al., 2006), the hurricane intensified in $10-12 \mathrm{~ms}^{-1}$ shear. There is also observational evidence (e.g. Black et al., 2002, Hurricane Jimena in 13$20 \mathrm{~ms}^{-1}$ shear) that TCs can remain intact in strong shear. These examples give us confidence that the resiliency of the TC is not a pathology of our numerical model or the employed parameterisations.

The vortex tilt in the shear experiments is significantly larger than in the no_shear case in the early evolution (Fig. 5). As can be expected, the vortex tilt increases with the magnitude of the shear. Later in the evolution, the tilt magnitude decreases in all experiments. After $24 \mathrm{~h}$ the tilt is about $5 \mathrm{~km}$ in the $10 \mathrm{mps}$ and $15 \mathrm{mps}$ cases, only slightly larger than in the no_shear case. In the $20 \mathrm{mps}$ case it takes longer (approx. $36 \mathrm{~h}$ ) for the vortex to reduce the tilt. Subsequently, tilt values larger than $10 \mathrm{~km}$ can still be found. For all cases, however, it is evident that the vortices are resilient to the detrimental effect of the shear and tend to re-align in the course of the experiment. The tilt evolution will be discussed further in Sect. 5.

\section{Thermodynamic impact of vertical shear on the boundary layer}

It will be shown in this section that the interaction with vertical shear has a profound impact on the thermodynamic structure of the BL. Downdrafts transport low $\theta_{e}$ air into the BL and form extended areas of depressed BL moist entropy, ultimately leading to a reduction of $\theta_{e}$ values within the eyewall updrafts. We will argue that this constitutes a frustration of the TC's energy cycle. The structure, magnitude, and evolution of the depressed BL $\theta_{e}$ will be documented and its impact on the azimuthal mean structure of the storm quantified. We will calculate the downward flux of low $\theta_{e}$ air into the BL and show that this downward flux is closely associated with the intensity evolution.

\subsection{Near-core entropy modification in the boundary layer}

\subsubsection{Banded structure of reduced $\theta_{\mathrm{e}}$}

An extensive region of considerably reduced $\mathrm{BL} \theta_{e}$ values can be found within a few hours after the vertical shear is imposed. This region of depressed $\mathrm{BL} \theta_{e}$ forms a quasistationary band that spirals inward from the Northwest and approaches the eyewall on the upshear side (East) of the storm (Fig. 7). The band can extend outwards up to 150$200 \mathrm{~km}$ radius. Air parcels with $\theta_{e}$ values between $351 \mathrm{~K}$ and $355 \mathrm{~K}$ could be advected inward from the environment. The high-amplitude localised minima, however, cannot be accounted for by horizontal advection because the minimum $\theta_{e}$ values are much smaller than that found in the environment at the same altitude ${ }^{6}$. The $\theta_{e}$ depression of these local minima is significant, with values of $\mathrm{BL} \theta_{e} 15-20 \mathrm{~K}$ lower than in the no_shear case ${ }^{7}$. The low $\theta_{e}$ values first appear to the Southwest of the storm centre (not shown). For higher shear values the $\mathrm{BL} \theta_{e}$ depression is evidently more pronounced.

In the 10mps case this spiral pattern lasts until $6 \mathrm{~h}$ and reappears from $14 \mathrm{~h}$ to $18 \mathrm{~h}$. In the $15 \mathrm{mps}$ and $20 \mathrm{mps}$ case the spiral pattern becomes somewhat less distinct after $7 \mathrm{~h}$ but low $\theta_{e}$ values still dominate the $\mathrm{BL}$ in the near-core region until $24 \mathrm{~h}$ and $36 \mathrm{~h}$, respectively. This extensive region of depressed $\mathrm{BL} \theta_{e}$ is associated with the pronounced intensity decrease after imposing the shear (cf. Fig. 3).

In the no_shear case there is an indication of a spiral pattern of depressed $\theta_{e}$ values also (Fig. 7a). Besides being far less pronounced, this spiral feature does not reach close to the eyewall and is a transient feature.

\subsubsection{Recovery of boundary layer $\theta_{\mathrm{e}}$}

After the widespread initial depression the BL $\theta_{e}$ values recover. The recovery process is illustrated for the $15 \mathrm{mps}$ and $20 \mathrm{mps}$ case in Fig. 8 by displaying two representative BL $\theta_{e}$ configurations, respectively. At $32 \mathrm{~h}$ the $\mathrm{BL} \theta_{e}$ values have recovered to a large degree in the $15 \mathrm{mps}$ case (Fig. 8a). Less pronounced bands of lower $\theta_{e}$ air (exemplified in Fig. 8b) infrequently interrupt the recovery process. The BL recovery in the $20 \mathrm{mps}$ case is much less complete. The $\mathrm{BL} \theta_{e}$ pattern rarely has a symmetric appearance, an exception is depicted in Fig. 8c. A band of low $\theta_{e}$ air spiraling cyclonically inward is found much more frequently (Fig. 8d), reminiscent of the initial pattern depicted in (Fig. 7d) though less pronounced. A more complete recovery is found only after $60 \mathrm{~h}$ (not shown). The slow and incomplete recovery of BL $\theta_{e}$ values in the $20 \mathrm{mps}$ case is consistent with the lower intensity and the delayed re-intensification of the storm. In the $10 \mathrm{mps}$ case the BL $\theta_{e}$ values have almost completely recovered at $20 \mathrm{~h}$ (not shown), consistent with the early re-intensification of the storm. Subsequently, areas of low $\operatorname{BL} \theta_{e}$ values are more confined and infrequent.

\footnotetext{
${ }^{6}$ Based on a preliminary analysis of the kinematics of the vortexshear flow, we offer an explanation for the interplay between horizontal and vertical advection in the authors' response to the anonymous reviewer (item 8), available at the ACPD webpage.

${ }^{7}$ This significant $\theta_{e}$ depression is associated with a $4-5 \mathrm{gkg}^{-1}$ depression in the water vapor mixing ratio and a $2-3 \mathrm{~K}$ depression in $\theta$ relative to the no_shear case. The spatial distribution of water vapor and $\theta$ at the time shown in Fig. 7 is presented in the authors' response to the anonymous reviewer (item 9) on the ACPD webpage.
} 
(a)
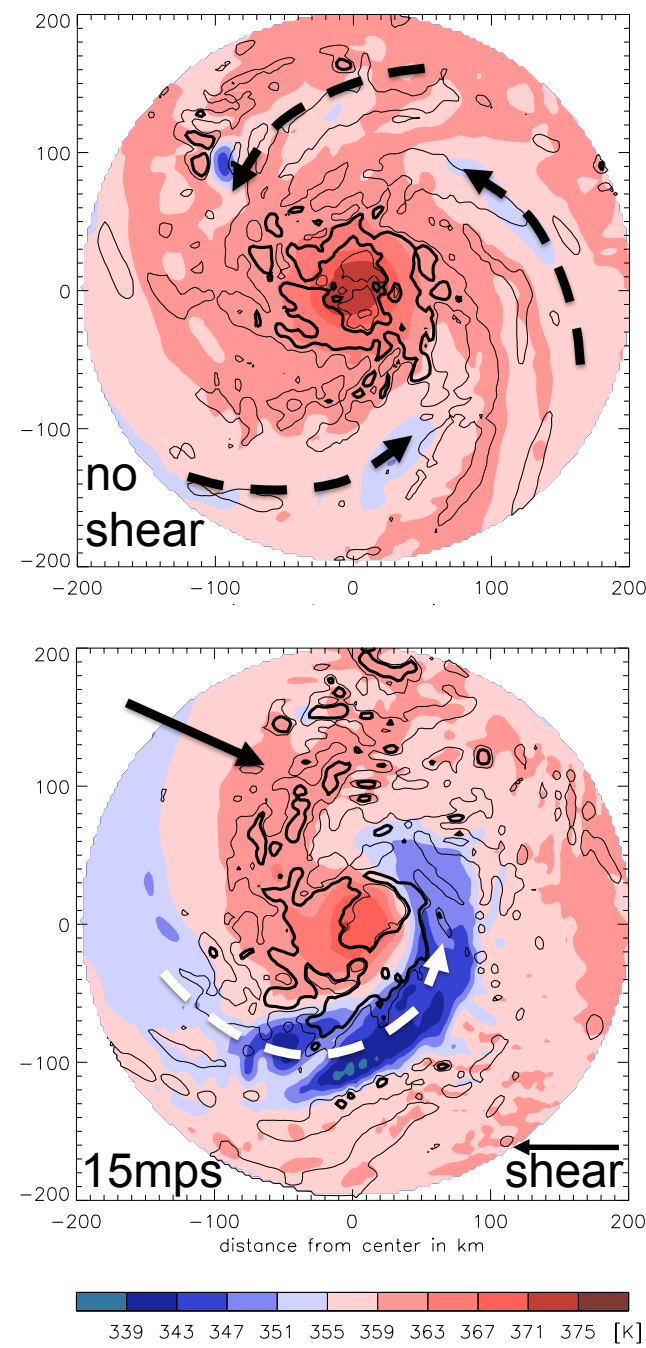

(b)
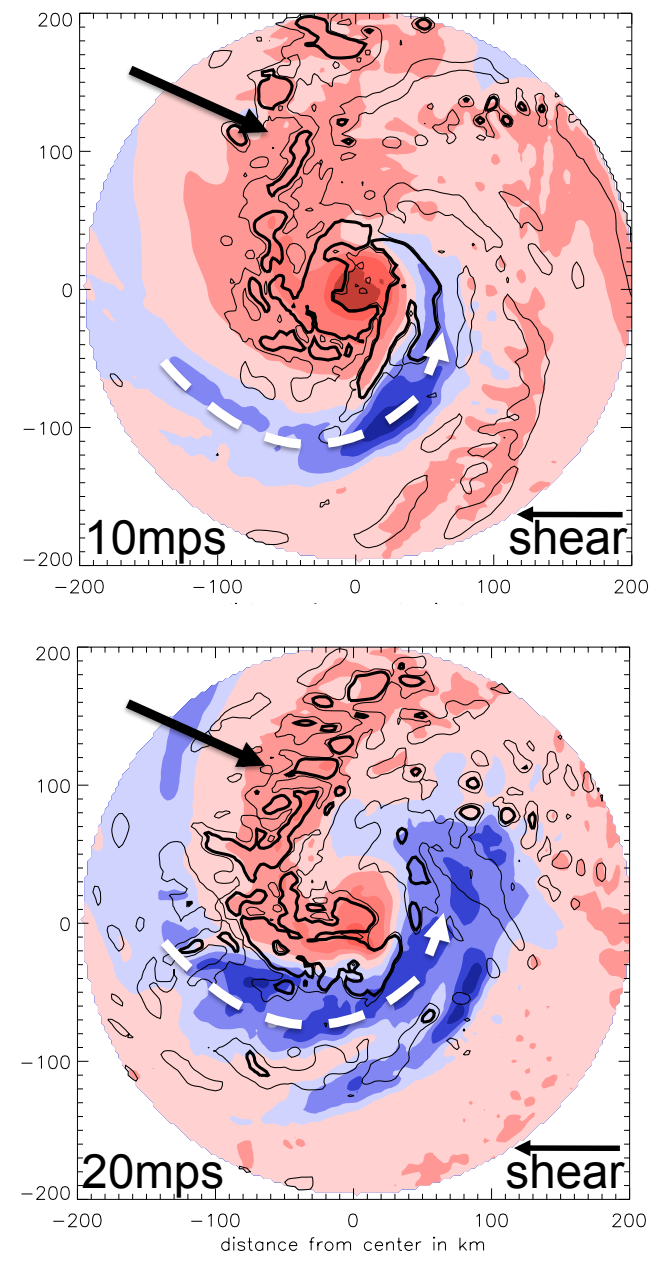

(d)
$339343347351355359363367371375[K]$

Fig. 7. Boundary layer $\theta_{e}$ (color, averaged over the lowest $1 \mathrm{~km}$ ), and upward motion (thin contour: $0.2 \mathrm{~ms}^{-1}$, thick contour: $1 \mathrm{~ms}^{-1}$, averaged between 1.25 and $2 \mathrm{~km}$ height) at $5 \mathrm{~h}$. The centre of the TC is in the middle of the domain. The no_shear case is depicted in (a), the 10mps, $15 \mathrm{mps}$, and $20 \mathrm{mps}$ case in (b), (c), and (d), respectively. The shear direction is indicated in the lower right corner of each plot. Solid arrows highlight the quasi-stationary convective asymmetry outside of the eyewall in the shear cases and dashed white arrows the quasi-stationary region of depressed $\mathrm{BL} \theta_{e}$ air. The dashed black arrows indicate transient bands of less-reduced $\theta_{e}$ values in the no_shear case. The depicted times are representative for the early part of the experiments (see text for details).

\subsubsection{A link to the convective asymmetry outside of the eyewall}

The $\mathrm{BL} \theta_{e}$ evolution is associated with a pronounced convective asymmetry outside of the eyewall. At early times in the shear experiments strong convective activity is found downshear-right of the centre (Fig. 7). The convection takes the form of bands and clusters that tend to rotate from the downshear-right into the downshear quadrant. This convective asymmetry is, in general, well pronounced until $30 \mathrm{~h}$ in the $15 \mathrm{mps}$ case. With the demise of the convective asymmetry the BL $\theta_{e}$ starts to recover (Fig. 8a). Subsequently, the convective asymmetry occurs infrequently, usually associated with a weak BL $\theta_{e}$ depression (e.g. Fig. 8b). In the $20 \mathrm{mps}$ case the pronounced convective asymmetry prevails until $60 \mathrm{~h}$ (e.g. Fig. 8d), interrupted by a short period of more axisymmetric structure around $46 \mathrm{~h}$ (Fig. 8c). Again, the more axisymmetric distribution of convection outside the eyewall is associated with a short period of recovered $\mathrm{BL} \theta_{e}$ values. In the $10 \mathrm{mps}$ case, the convective asymmetry exhibits a minimum around $12 \mathrm{~h}$ and is temporarily reestablished afterwards (not shown). After $24 \mathrm{~h}$, a pronounced and quasistationary asymmetry is no longer found.

In all of our experiments, a depression of $\mathrm{BL} \theta_{e}$ in general occurs in association with a quasi-stationary convective asymmetry outside of the eyewall. Section 5 will discuss the generation and the importance of this convective asymmetry in further detail. 
(a)
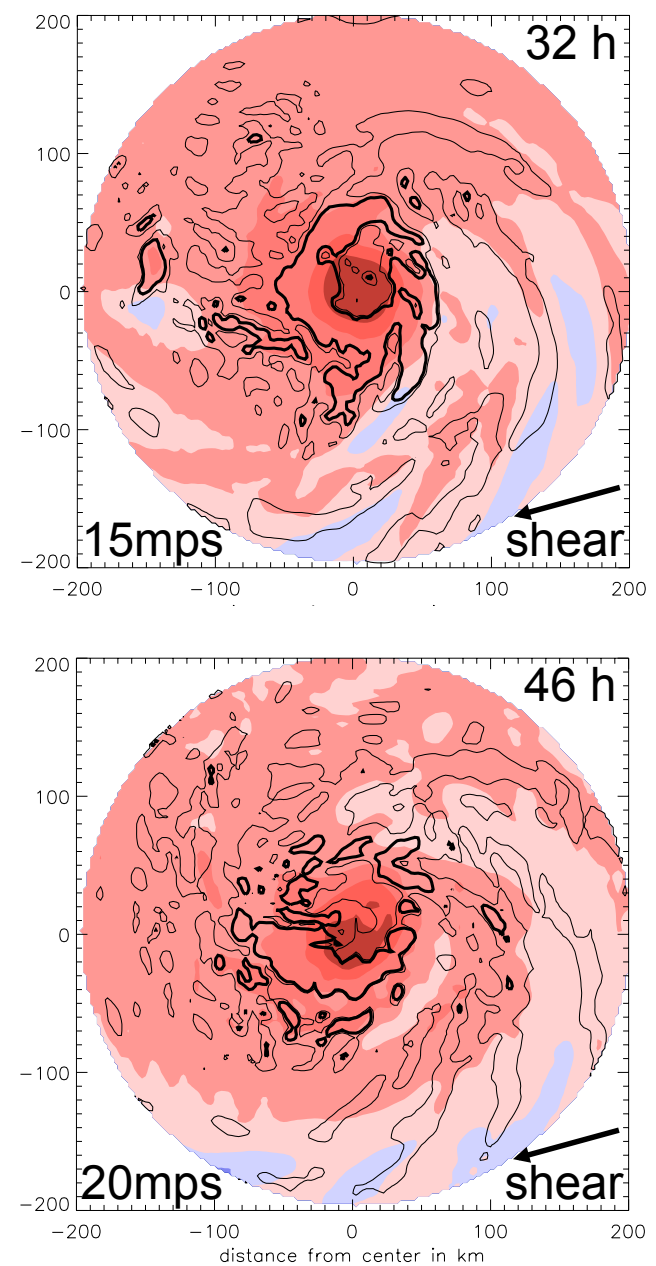

(c)

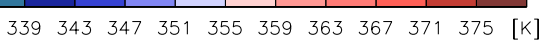

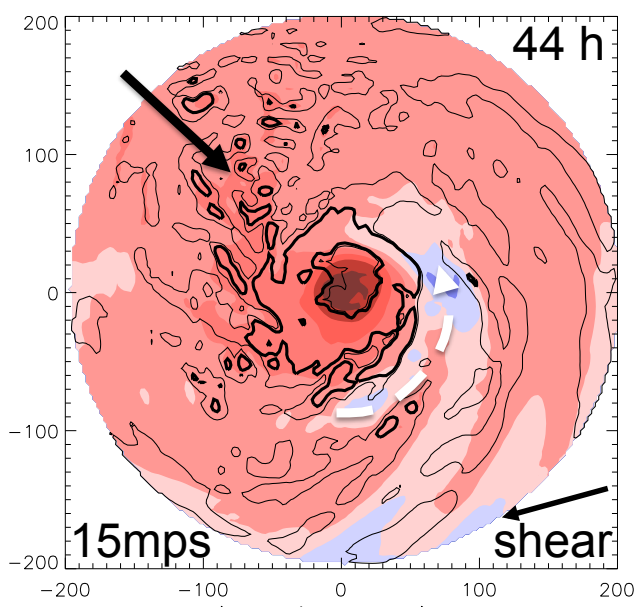

(b)

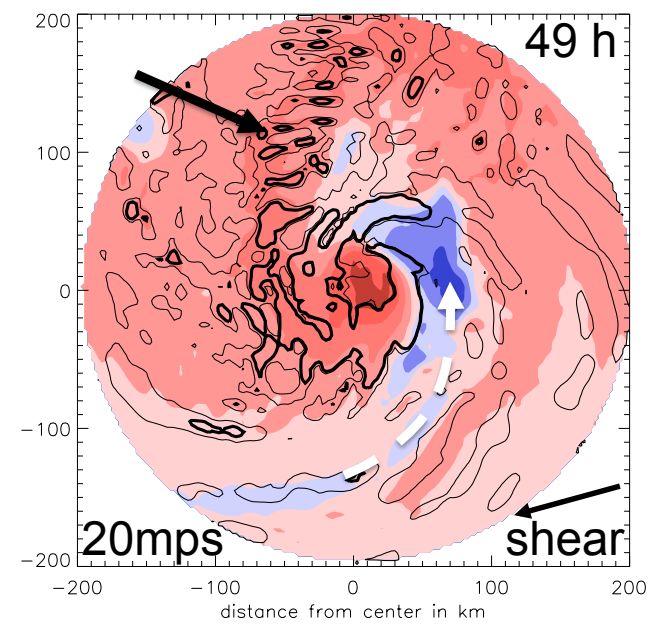

(d) $\begin{array}{lllllllllll}339 & 343 & 347 & 351 & 355 & 359 & 363 & 367 & 371 & 375 & {[\mathrm{~K}]}\end{array}$

Fig. 8. Same as Fig. 7, but for the $15 \mathrm{mps}$ case (top row) at times $32 \mathrm{~h}$ (a), and $44 \mathrm{~h}$ (b) and for the $20 \mathrm{mps}$ case (bottom row) at times $46 \mathrm{~h}$ (c), $49 \mathrm{~h}$ (d).

\subsubsection{Impact on the azimuthal mean structure}

The $\operatorname{BL} \theta_{e}$ depression is clearly a spiral-like feature with a strong wave number 1 structure. A theory for TC intensity, however, only exists for steady-state axisymmetric storms. We note that an axisymmetric theory is likely an oversimplification for a strongly asymmetric TC. Further progress needs to be made in our theoretical understanding of asymmetric storms to tackle the important forecast problem of vertical shear impacts on storm intensity. Lacking a more complete theoretical foundation we present in this subsection an axisymmetric view of the frustration of the TC power engine.

Figure 9 depicts the vertical and radial distribution of azimuthally-averaged $\theta_{e}$ in the lower part of the TC at the same early time as in Fig. 7. The same general structure is found in all shear cases: a tongue of low $\theta_{e}$ air effectively intrudes into the BL and radially inward towards the eyewall updrafts (Sect. 4.2). This apparent intrusion is realised in highly asymmetric downdrafts while the contribution from the mean descent is very small. The $\theta_{e}$ depression is most pronounced between $50 \mathrm{~km}$ and $100 \mathrm{~km}$ radius. As can be expected from the horizontal plots in Fig. 7 this tongue of low $\theta_{e}$ is more pronounced for higher shear values. A qualitatively similar vertical structure of the azimuthally averaged $\theta_{e}$ distribution is also found at subsequent times when bands of decreased BL $\theta_{e}$ values are present (e.g. at the times depicted in Figs. 8b, and 8d, vertical cross sections not shown). The apparent intrusion of low $\theta_{e}$ air at these times is less pronounced and confined closer to the eyewall.

We can estimate a representative $\theta_{e}$ value of air rising in the eyewall by visually averaging the $\theta_{e}$ values within the $1 \mathrm{~ms}^{-1}$ updrafts in Fig. 9. Compared to the no_shear case, we find that the $\theta_{e}$ is lowered by approximately $9 \mathrm{~K}, 4 \mathrm{~K}$, and $3 \mathrm{~K}$ in the $20 \mathrm{mps}, 15 \mathrm{mps}$, and $10 \mathrm{mps}$ case, respectively. 


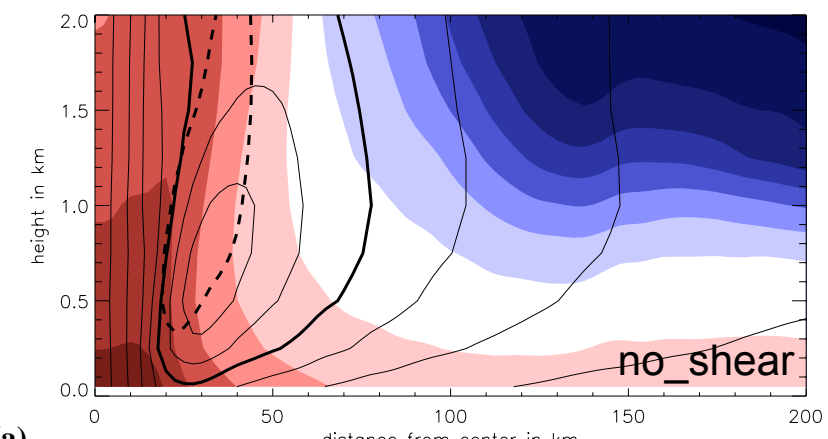

(a)

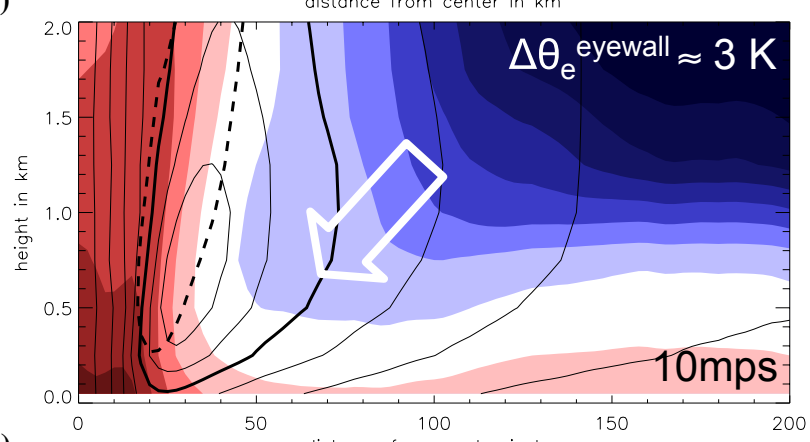

(b)

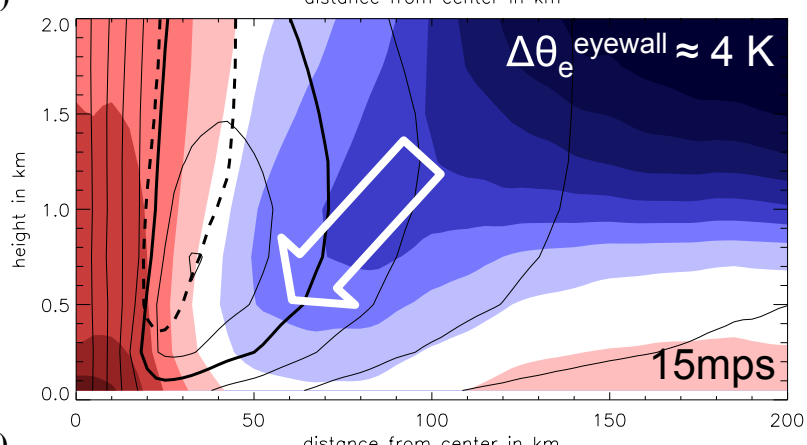

(c)

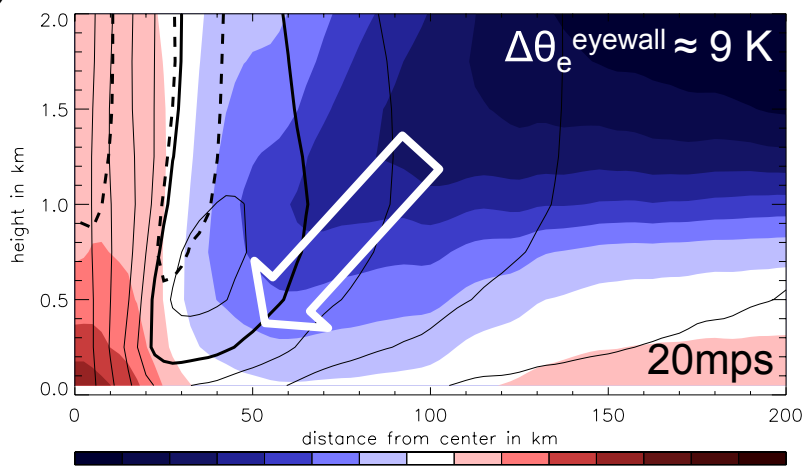

(d)

$336339342345348351354357360363366369372375[\mathrm{~K}]$

Fig. 9. Azimuthal mean of $\theta_{e}$ (color), tangential wind (solid contours, every $10 \mathrm{~ms}^{-1}, 50 \mathrm{~ms}^{-1}$ thick), and vertical motion (dashed, every $1 \mathrm{~ms}^{-1}$ ) displayed in a height-radius format for the no_shear (a), 10mps (b), $15 \mathrm{mps}$ (c), and $20 \mathrm{mps}$ case (d) at $5 \mathrm{~h}$, representative for the early part of the experiments. The white arrows highlight the apparent intrusion of a tongue of low $\theta_{e}$ air into the BL. The lenght of the arrow is scaled by $\Delta \theta_{e}^{\text {eyewall }}$, the $\theta_{e}$ reduction in the eyewall as compared to the no_shear case (see text for details).
The reduction of eyewall $\theta_{e}$ at this time clearly precedes the subsequent intensity evolution: Under no-shear conditions the storm intensifies in the following $12 \mathrm{~h}$ to $87 \mathrm{~ms}^{-1}$, the intensification in the $10 \mathrm{mps}$ case is significantly less and the TCs in the $15 \mathrm{mps}$ and $20 \mathrm{mps}$ cases both weaken (cf. Fig. 3).

A trajectory analysis of Cram et al. (2007) found a decrease of eyewall $\theta_{e}$ by entrainment of environmental air at mid-levels of $\mathcal{O}(1) \mathrm{K}$ in a full physics model simulation of a mature, moderately sheared $\left(12 \mathrm{~ms}^{-1}\right)$ hurricane. A comparison with the values above suggests that BL processes dominate the intensity evolution in our numerical experiment and that mid- and upper level processes are of secondary importance.

Moreover, the radial profile of the azimuthal mean tangential winds at $2 \mathrm{~km}$ (the upper bound in Fig. 9) is similar in all cases, while the wind maximum between $500 \mathrm{~m}$ and $1 \mathrm{~km}$ has already decreased by 7 and $15 \mathrm{~ms}^{-1}$ in the $15 \mathrm{mps}$ and $20 \mathrm{mps}$ case, respectively. The vortex evidently weakens first at the lowest levels, as opposed to a "top-down" decay. This finding supports our hypothesis that low-level processes - the depression of BL entropy by shear-induced downdrafts - dominate over possible processes operating at mid- to upper-level.

\subsection{Downward flux of low $\theta_{\mathrm{e}}$ air into the boundary layer}

The vertical structure of the azimuthal mean $\theta_{e}$ field as well as the temporal evolution of the asymmetric $\operatorname{BL} \theta_{e}$ depression offer evidence that low $\theta_{e}$ air is brought into the BL from above $^{8}$. This downward import is now examined in more detail.

\subsubsection{A definition of downward flux}

Here we define the downward flux $\theta_{e}$ at the top of the BL as

$\mathrm{DFX} \equiv w_{-} \theta_{e}^{\prime}$,

where $w_{-}$is negative vertical motion and the prime denotes the deviation from the azimuthal mean. The top of the BL is defined here as $1.5 \mathrm{~km}$, roughly the top of the axisymmetric inflow layer. Positive values of DFX denote downward flux of anomalously low $\theta_{e}$ into the BL (hereafter referred to as low $\theta_{e}$ flux). The definition of DFX differs from the vertical eddy flux of $\theta_{e}\left(w^{\prime} \theta_{e}^{\prime}\right)$ in that the latter expression yields positive values for upward flux of positive $\theta_{e}$ anomalies out of the BL also. Downward flux of negative $\theta_{e}^{\prime}$ and upward flux of positive $\theta_{e}^{\prime}$ can therefore not be distinguished. Outside of the eyewall, mean vertical motion $\bar{w}$ is very small $\left(\mathcal{O}\left(1 \mathrm{cms}^{-1}\right)\right)$ and vertical motion is dominated by the asymmetries. In this case, $\mathrm{DFX} \approx w_{-}^{\prime} \theta_{e}^{\prime}$, where $w_{-}^{\prime}$ denotes negative anomalies. The horizontal distributions of $w_{-}^{\prime} \theta_{e}^{\prime}$ and DFX are similar and the azimuthal averages, $\overline{\mathrm{DFX}}$ and $\overline{w_{-}^{\prime} \theta_{e}^{\prime}}$, are virtually the same (not shown). Because the total vertical motion could

\footnotetext{
${ }^{8}$ Non-pseudoadiabatic processes, e.g. precipitative flux, are arguably of secondary importance for the depression of $\operatorname{BL} \theta_{e}$ values.
} 
(a)
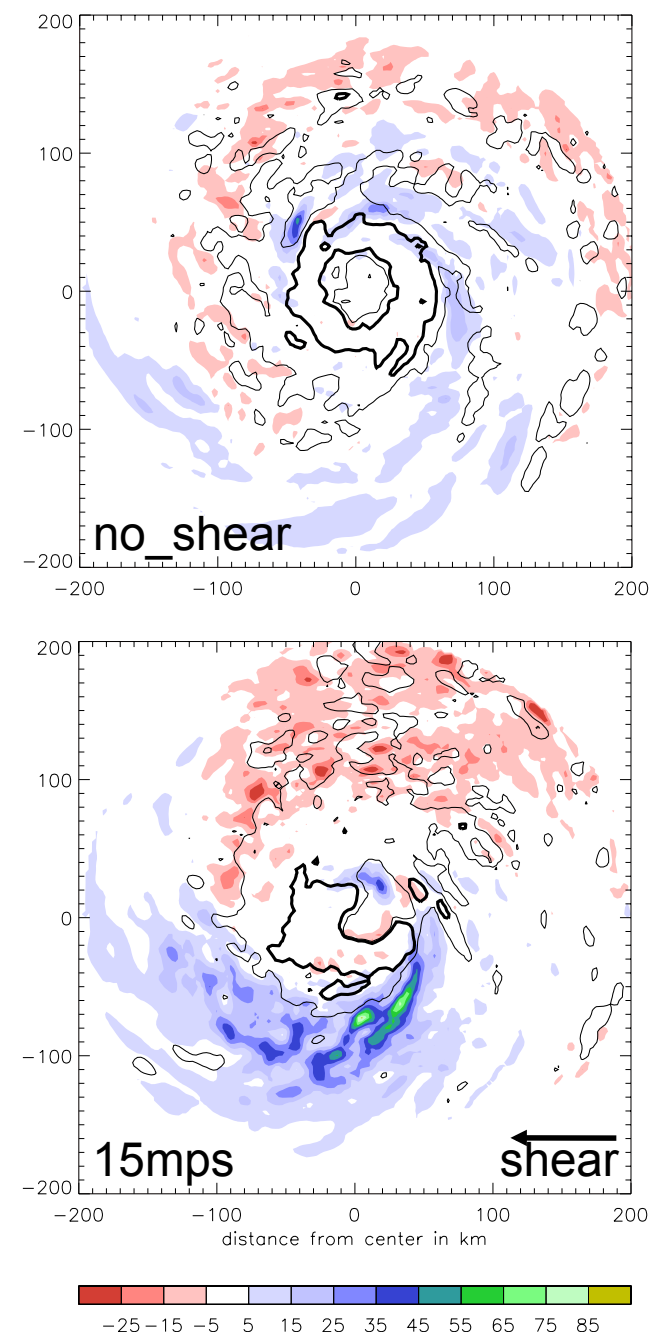

(b)
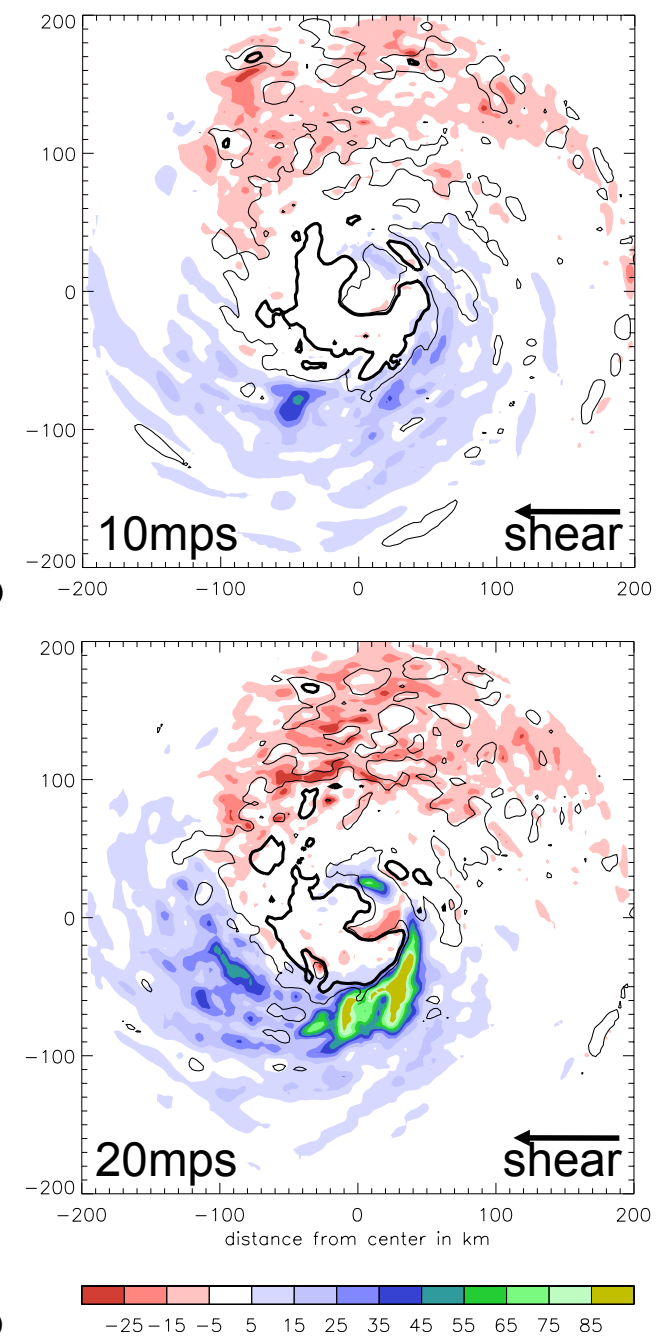

Fig. 10. Same as Fig. 7, but for the downward flux of $\theta_{e}^{\prime}$, DFX (color, in $0.1 \mathrm{Kms}^{-1}$ ), at the top of the inflow layer, defined as $1.5 \mathrm{~km}$, averaged from $1 \mathrm{~h}$ to $6 \mathrm{~h}$. The no_shear case is depicted in (a), the 10mps, 15mps, and 20mps cases are depicted in (b), (c), and (d), respectively.

be positive even when $w^{\prime}<0$ (for $\bar{w}>\left|w_{-}^{\prime}\right|$ ), and since we are interested in air parcels that indeed penetrate into the BL from above, we prefer the DFX metric (Eq. 23).

\subsubsection{Downward flux pattern and its evolution}

The downward flux DFX, averaged over the first $6 \mathrm{~h}$ of shear, is shown in Fig. 10. At this early time the downward flux pattern matches very well the structure of low $\theta_{e}$ values in the BL. Downward flux of low $\theta_{e}$ air is found in a banded region extending from $\approx 200 \mathrm{~km}$ radius West (downshear) of the centre to close to the eyewall on the East (upshear) side. The downward flux of low $\theta_{e}$ is more pronounced for stronger shear values. Comparing Fig. 7 and Fig. 10 it is clearly evident that low BL $\theta_{e}$ air is advected horizontally by the swirling winds also, in particular in the $15 \mathrm{mps}$ and $20 \mathrm{mps}$ case, extending the area of depressed BL entropy into the northeastern quadrant of the storm.
After $5 \mathrm{~h}$, a characteristic value of the $\theta_{e}$ depression in the $15 \mathrm{mps}$ case is $15 \mathrm{~K}$ at $60 \mathrm{~km}$ radius. Assuming an azimuthal wind of $50 \mathrm{~ms}^{-1}$, an air parcel circumvents the centre at this radius in $\approx 2 \mathrm{~h}$. Significant DFX values are found approximately in a semicircle around the centre. A BL air parcel thus remains in an area of downward flux of low $\theta_{e}$ for $\approx 1 \mathrm{~h}$. For the $15 \mathrm{mps}$ case we estimate an average DFX value of $3 \mathrm{Kms}^{-1}$. To cool a BL column of $1 \mathrm{~km}$ by $15 \mathrm{~K}$ it thus takes $\approx 1.5 \mathrm{~h}$. This short time scale, in particular as we have neglected the replenishment of $\theta_{e}$ by surface fluxes, is consistent with the occurrence of the $\operatorname{BL} \theta_{e}$ depression soon after the shear is imposed. This estimate and the spatial patterns shown in Fig. 7 and Fig. 10 offer strong evidence that the BL $\theta_{e}$ depression is caused by downward flux of low $\theta_{e}$ air into the BL. 


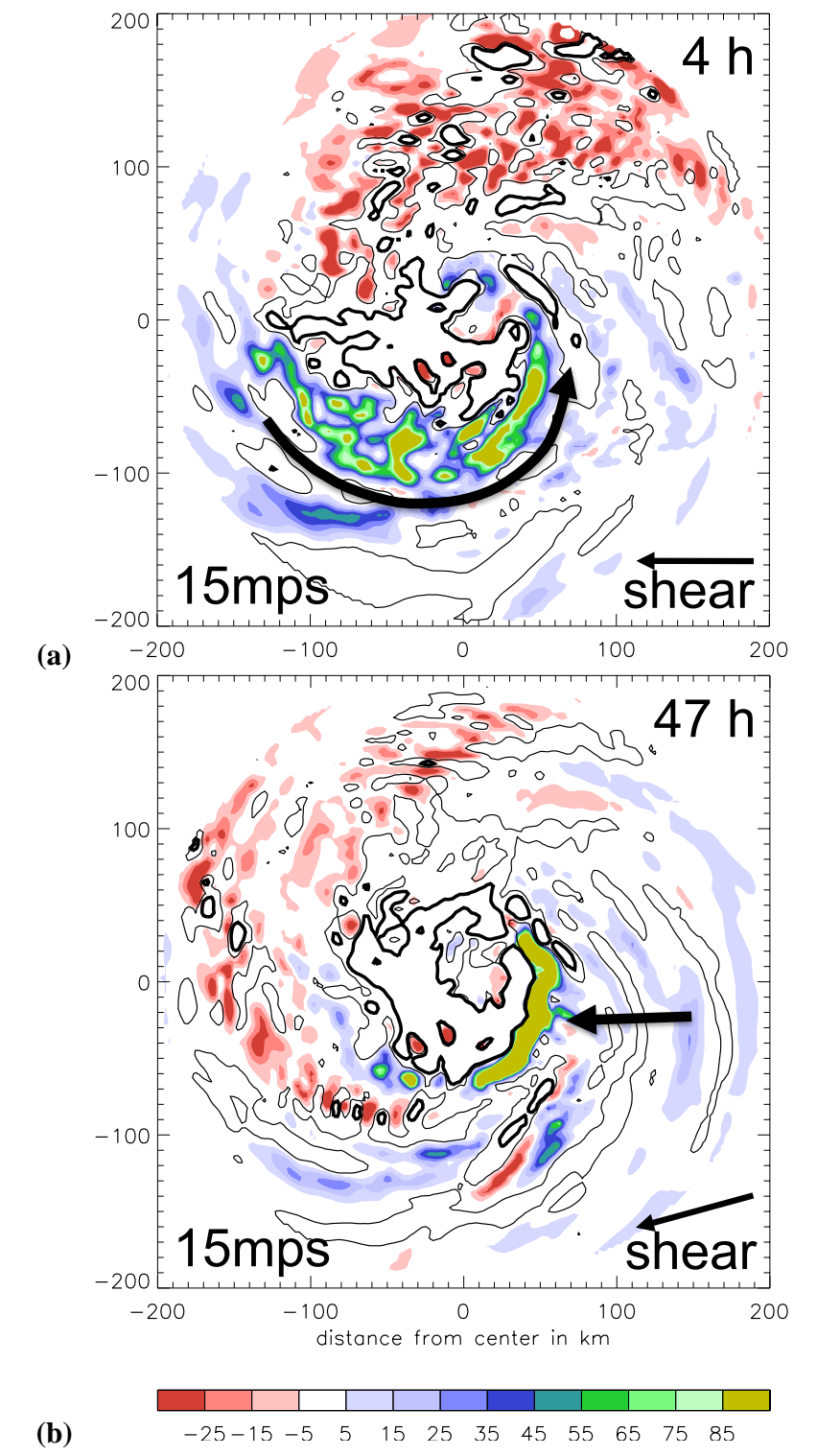

Fig. 11. Same as Fig. 10, but for the $15 \mathrm{mps}$ case at times $4 \mathrm{~h}$ (a) and $47 \mathrm{~h}$ (b). The thick black arrows highlight the characteristic structure of DFX at the respective time.

The instantaneous DFX distribution for the $15 \mathrm{mps}$ case is illustrated in Fig. 11. The instantaneous field exhibits a pronounced quasi-stationary banded structure in the first $\approx 24 \mathrm{~h}$ after the shear is imposed (exemplified in Fig. 11a), however with a notable variability in magnitude. The orientation of the band shifts slightly counterclockwise (not shown), extending southwest to northeast, consistent with the gradual increase of the northerly shear component (see Sect. 6). From $24-48 \mathrm{~h}$ the DFX pattern is less distinct and a pronounced band of downward fluxes occurs infrequently (not shown). After $48 \mathrm{~h}$ the downward flux is found to occur predominantly on the upshear side of the storm, adjacent to the eyewall (Fig. 11b). The 10mps case shows very similar characteristics, albeit with smaller magnitude (not shown). In the 20mps case the banded structure dominates the DFX pattern until $\approx 48 \mathrm{~h}$ and subsequently, pronounced bands still occur frequently (not shown).

In the no_shear case bands of downward flux of low $\theta_{e}$ are also evident. However, these bands are of smaller radial extent, the magnitude of downward transport is much less and, in particular, they rotate around the vortex and thus do not exhibit the quasi-steady location as their counterparts in the sheared cases do. The largest values of downward flux in the no_shear case are in general found adjacent to the eyewall. An example of the transient nature of the DFX pattern in the no_shear case is given in Fig. 12. The sudden introduction of shear constitutes an artificial impact on the TC. The $\theta_{e}$ fluxes, however, show coherent temporal and spatial structure in the shear experiments. We thus believe that imposing the shear in an instantaneous - and thus rather unrealistic - manner should not invalidate the use of our model results for the real atmosphere.

\subsection{Intensity modification and downward flux of $\theta_{\mathrm{e}}$}

Consistent with our examination of the azimuthal mean $\theta_{e}$ structure we now consider the azimuthally averaged downward flux of $\theta_{e}, \overline{\mathrm{DFX}}$. For the azimuthal average we can examine the time evolution of the magnitude and the radial distribution of the $\theta_{e}$ flux in a compact manner and compare it with the intensity evolution (Fig. 13).

Inspection of Fig. 13 shows that the overall magnitude of the downward flux increases with higher shear values. In the no_shear case (Fig. 13a) the downward flux of low $\theta_{e}$ air is mostly confined to within $80 \mathrm{~km}$ of the storm centre. It is interesting to note that the downward flux of low $\theta_{e}$ air near the eyewall becomes a persistent feature after the storm has reached its quasi-equilibrium intensity of $100 \mathrm{~ms}^{-1}$ at $\approx 45 \mathrm{~h}$. In the shear experiments not only the magnitude of the downward flux is greater but the radial extent is larger than in the no_shear case also. Bands of downward flux frequently extend out to a radius of $140 \mathrm{~km}-150 \mathrm{~km}$. A much larger area of the BL is thus affected by downward flux of low $\theta_{e}$ in the shear cases. The largest values are found outside the radius of maximum winds (RMW) between $40 \mathrm{~km}$ and $80 \mathrm{~km}$ radius. Considerably smaller values are found within the RMW.

The initial weakening of intensity in the shear cases is clearly associated with enhanced low $\theta_{e}$ flux into the BL. This initial flushing of the BL with low $\theta_{e}$ air lasts for about $6 \mathrm{~h}, 12 \mathrm{~h}$, and $24 \mathrm{~h}$ in the $10 \mathrm{mps}, 15 \mathrm{mps}$, and $20 \mathrm{mps}$ case, respectively. After this initial period, the intensity evolution is closely associated with $\overline{\mathrm{DFX}}$ on the time scale of a few hours. Periods of re-intensification are usually associated with small values of $\overline{\mathrm{DFX}}$, whereas high values of $\overline{\mathrm{DFX}}$ most often precede periods of weakening or an interruption in reintensification. We have highlighted some illustrative downward flux events with light gray arrows in Fig. 13. In the 
(a)

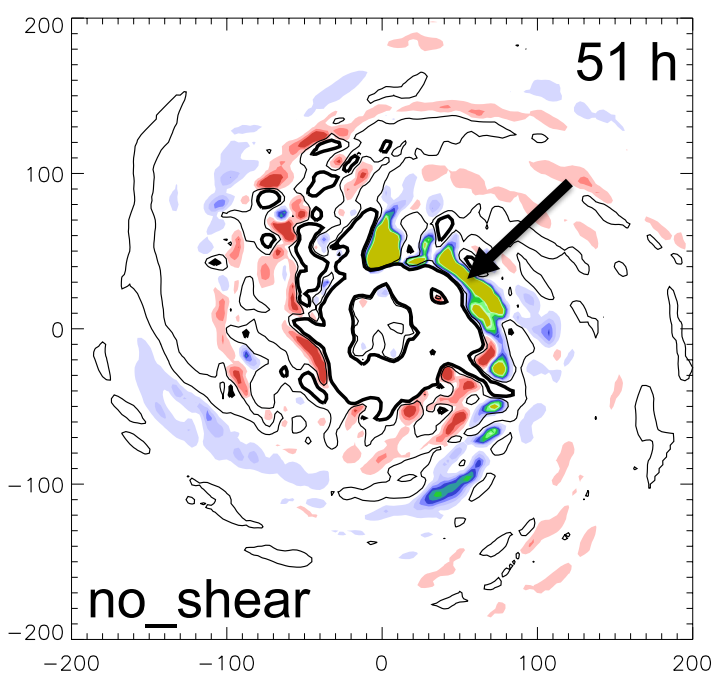

(b)
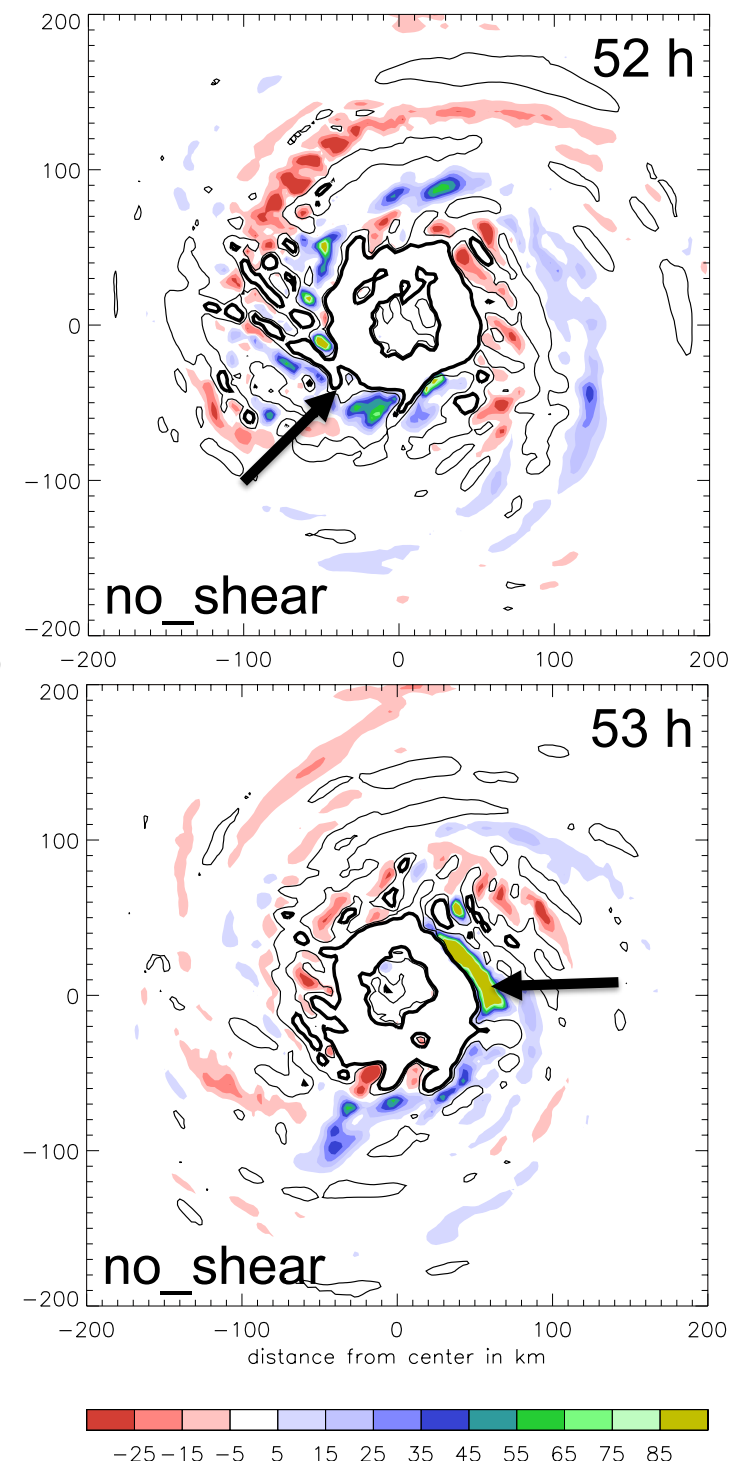

Fig. 12. Same as Fig. 10, but for the no_shear case at 3 consecutive hours (51 h (a), $52 \mathrm{~h} \mathrm{(b),} \mathrm{and} 53 \mathrm{~h} \mathrm{(c))}$. 10mps case (Fig. 13b) there is a close relation between $\overline{\mathrm{DFX}}$ and intensity at $13 \mathrm{~h}, 35 \mathrm{~h}, 45 \mathrm{~h}$, and $68 \mathrm{~h}$. In the $15 \mathrm{mps}$ case (Fig. 13c) the flux events at $19 \mathrm{~h}$ and $26 \mathrm{~h}$ frustrate the reintensification of the storm. During the subsequent period of relatively small values of $\overline{\mathrm{DFX}}$ intensity increases from $75 \mathrm{~ms}^{-1}$ to $90 \mathrm{~ms}^{-1}$. A local intensity minimum around $40 \mathrm{~h}$ is associated with enhanced downward flux. The storm does not intensify above $90 \mathrm{~ms}^{-1}$ before a period of small $\overline{\mathrm{DFX}}$ values starts at $53 \mathrm{~h}$. The $7 \mathrm{~ms}^{-1}$ weakening late in the experiment can be attributed to a flux maximum at $65 \mathrm{~h}$. In the 20mps case (Fig. 13d) periods of strong fluxes of low $\theta_{e}$ air into the BL frustrate the re-intensification of the storm around $28 \mathrm{~h}, 40 \mathrm{~h}, 48 \mathrm{~h}, 53 \mathrm{~h}, 59 \mathrm{~h}$, and $63 \mathrm{~h}$. In the no_shear case, maxima in $\overline{\mathrm{DFX}}$ at $18 \mathrm{~h}$ and $33 \mathrm{~h}$ precede distinct local minima in intensity.

The close association of downward flux of low $\theta_{e}$ air into the $\mathrm{BL}$ and the intensity evolution adds support to the tenet that the depression of the near-core $\mathrm{BL} \theta_{e}$ plays a significant role in the intensity modification of the sheared TC. The radial-height cross sections (Fig. 9) have demonstrated that, arguably by the radial advection in the inflow layer, the lower BL $\theta_{e}$ values outside the eyewall lead to a significant decrease of axisymmetric $\theta_{e}$ values in the eyewall updrafts also. A significant impact of shear pertinent to intensity modification is thus a frustration of the energy cycle of the storm in the inflow layer.

We note that in the presence of pronounced vortex-scale asymmetries the applicability of an axisymmetric viewpoint is questionable. Future work should be directed to better understand the influence of the asymmetric TC structure, in particular the asymmetric $\mathrm{BL} \theta_{e}$ depression and asymmetric inner core updrafts, on the frustration of the TC power machine in a vertically sheared TC.

\section{Dynamical origin of the boundary layer $\theta_{\mathrm{e}}$ depression}

In the previous section, we have presented evidence that the intensity modification of our sheared TCs is tied to the depression of the near-core BL $\theta_{e}$ caused by downward flux of low $\theta_{e}$ air. We will show now that the downward flux is due to downdrafts associated with a quasi-stationary convective asymmetry outside of the eyewall. This convective asymmetry, as we will argue, has its origin in the asymmetric balanced dynamics of the TC.

Only a brief assessment of the vortex dynamics is given here but a detailed investigation is planned for a forthcoming paper. The upshot of this is that the tilt of the sheared TCs in our warm-rain experiments, including the tilt of the outer vortex at larger radii, can be approximated to zero order by a standing wave number 1 VRW pattern.

Our focus here will be on the $15 \mathrm{mps}$ case. The features to be discussed have essentially the same characteristics in the $10 \mathrm{mps}$ and $20 \mathrm{mps}$ case. 
(a)
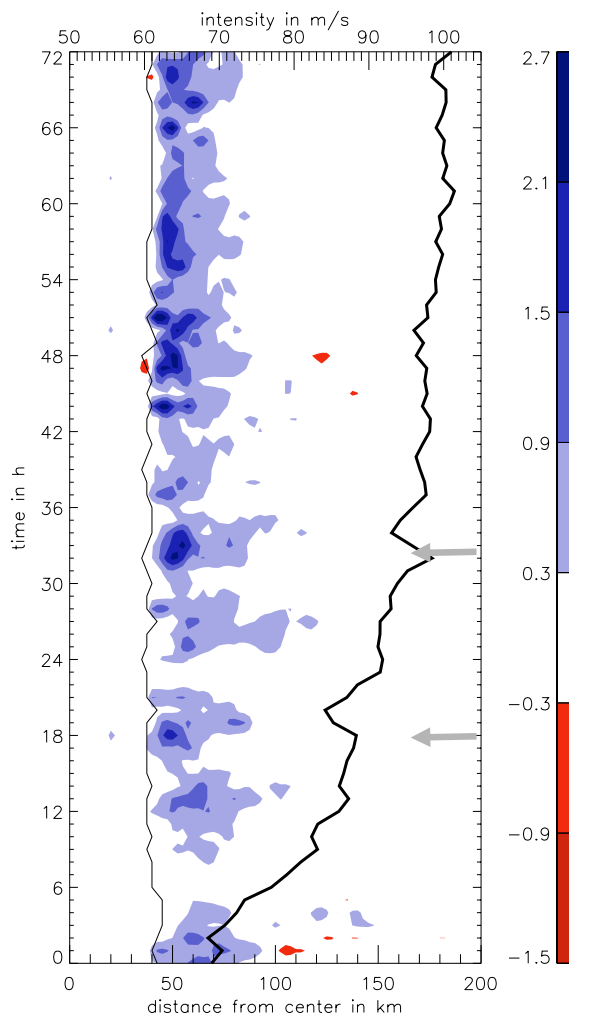

(b)
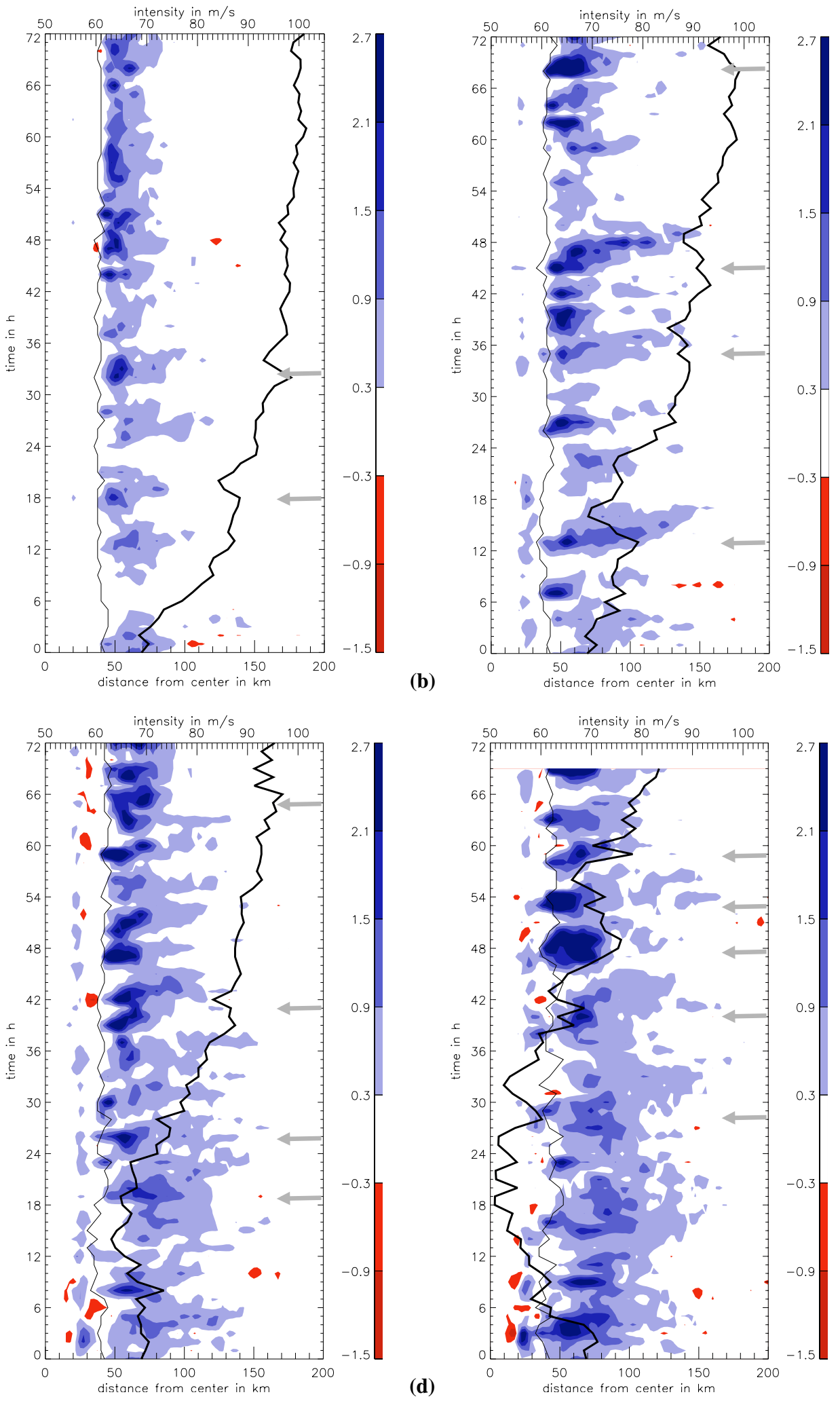

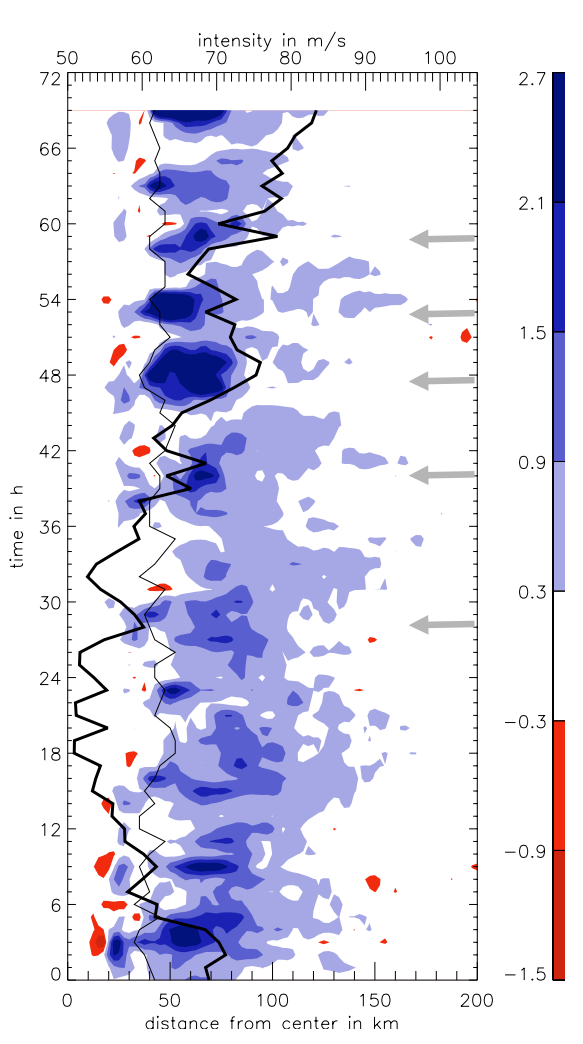

Fig. 13. Radial-time plot of the azimuthal average of downward flux of $\theta_{e}\left(\overline{\mathrm{DFX}}\right.$, see text for definition) at $1.5 \mathrm{~km}$ height (color, in $\left.\mathrm{Kms}^{-1}\right)$, time series of intensity (thick contour, scale at the top of the figures) and RMW (thin contour). The no_shear case is depicted in (a), the 10mps, 15mps, and 20mps case in (b), (c), and (d), respectively. Light gray arrows highlight DFX events that frustrate the re-intensification of the storm. 


\subsection{Is response to shear forcing dominated by balanced dynamics?}

Jones (1995) considered the evolution of an initially barotropic and dry TC-like vortex in terms of the formation and subsequent interaction of PV anomalies. Due to the differential advection associated with the vertical shear, a positive PV anomaly forms upshear at low-levels and downshear at upper-levels. These anomalies "induce" a quasi-horizontal circulation and their mutual advection leads to a cyclonic rotation of the tilt axis. Left of shear, this mutual advection and the differential shear advection can cancel and an equilibrium tilt direction is possible. This vortex evolution can also be explained in terms of VRW dynamics (Reasor et al., 2004). The perturbation of a vortex by shear strongly projects onto a vertical and azimuthal wave number 1 VRW. Reasor et al. have shown that, for a shear profile matching the vertical wave number 1 perturbation, the vortex dynamics can be described elegantly as a forced harmonic oscillator with damping. This model predicts that the vortex tilt asymptotes into the quasi-equilibrium configuration that is left of the shear vector while the initial vortex tilt decreases. Schecter and Montgomery (2007) have presented an alpha model for including the principal effects of moist processes on VRWs. This model predicts that cloud coverage will significantly increase the damping rate and thus increase the resiliency of the vortex. The theory has not yet been extended to account for the secondary circulation of a TC.

The TC in our experiment exhibits baroclinic structure and a pronounced secondary circulation, and unbalanced motion is fully accounted for in the numerical model. Despite these complexities, it is nonetheless remarkable that the tilt evolution of the sheared TC in our experiment is consistent with the predictions of the dry, balanced theory of Reasor et al., as we will demonstrate below.

\subsubsection{Some details on the tilt evolution of the modeled TC}

As discussed in Sect. 3, all of the sheared TCs in our experiment are resilient and reduce their tilt with time. We now revisit the tilt evolution and consider the tilt direction also. The tilt rapidly settles in a southern direction and only small oscillations around this equilibrium direction appear in the first $18 \mathrm{~h}$ (Fig. 14). While the tilt direction stays in the equilibrium direction, the tilt magnitude decreases slowly in time (Fig. 5), qualitatively consistent with the prediction of the forced-damped harmonic oscillator model of Reasor et al.. The decrease of vortex tilt during this time can thus be regarded as the manifestation of inviscid VRW damping of the tilt mode. A comparison of vertical cross-sections of the total vorticity field and the azimuthal wave number 1 field along the tilt axis suggests that indeed much of the total tilt structure is captured by wave number 1 (not shown). After the tilt magnitude has decreased significantly around $18 \mathrm{~h}$ the

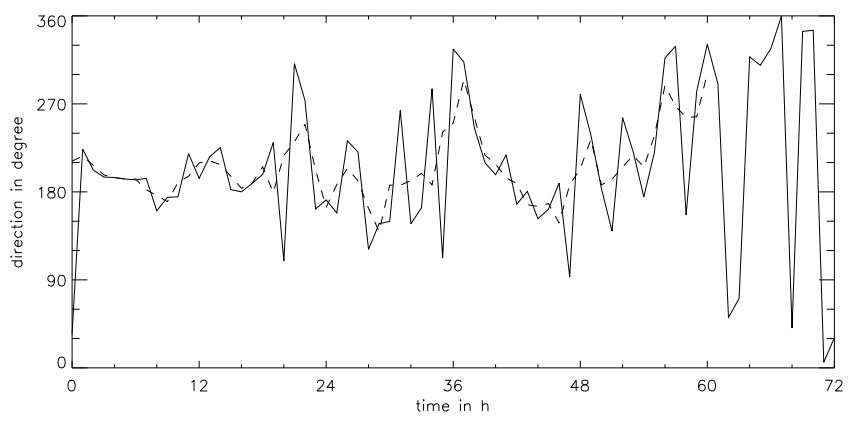

Fig. 14. Direction of tilt with height (solid) for the $15 \mathrm{mps}$ case $\left(0^{\circ}\right.$ and $360^{\circ}=$ North, $90^{\circ}=$ East, $180^{\circ}=$ South, and $270^{\circ}=$ West $)$. Tilt is calculated as the vector difference between the vorticity centroids at $10 \mathrm{~km}$ and $1 \mathrm{~km}$. The $6 \mathrm{~h}$ running mean (dashed) is shown up to $60 \mathrm{~h}$ also. Later in the experiment, it is not clear whether the TC vortex precesses with high frequency or oscillates around a predominant northerly direction. The averaged time series is thus shown until 60 h only.

oscillation of the vortex starts to deviate from the equilibrium direction. A higher frequency precession is then indicated in Fig. 14. The precession frequency is in the range of the precession frequency of the vortex in the no_shear case (see Sect. 3.1). The tilt magnitude after $18 \mathrm{~h}$ is only slightly greater than in the no_shear case.

\subsubsection{The guiding hand of balanced, wave number 1 asymmetries}

Using a Fourier analysis, we have confirmed that wave number 1 is by far (65-90\%, not shown) the dominant contribution to the total asymmetry of the tangential wind and the pressure fields ${ }^{9}$. The horizontal structure above the BL is well represented by wave number 1 (not shown). The ratio of the wave number 1 perturbation and the azimuthal mean is small $(0.05-0.2$, not shown).

The power spectra of vorticity and vertical motion are significantly flatter than for pressure and horizontal winds (not shown). This can be expected as both fields are highly coupled to convective processes that occur preferably at smaller scales. For both of these fields the wave number 1 asymmetry is not small compared to the respective azimuthal mean field. The wave number 1 pattern of these fields, however, forms an envelope in which most of the smaller scale asymmetries reside. This notion is illustrated for the vorticity field at a representative time in Fig. 15a.

\footnotetext{
${ }^{9}$ The values for the radial wind are essentially the same but the perturbation is not small compared to the azimuthal mean of the radial velocity.
} 


\subsubsection{A preliminary balance assessment}

For the sheared TCs in our experiments, the asymmetric structure is influenced strongly by the wave number 1 asymmetry out to radii of $150 \mathrm{~km}-200 \mathrm{~km}$. It will be shown now that this quasi-stationary wave number 1 asymmetry is largely in asymmetric balance (AB) (Shapiro and Montgomery, 1993).

For nearly steady flow asymmetries the $\mathrm{AB}$ expansion parameter is defined in the sense of a local Rossby number for azimuthal wave number $n$,

$R_{l}^{2}=\frac{n^{2} \bar{v}^{2} / r^{2}}{\bar{\eta} \bar{\xi}}$

with the mean tangential velocity $\bar{v}$, radius $r$, mean absolute vorticity $\bar{\eta}$, the local Coriolis parameter $\xi=f+2 v / r$, and the standard Coriolis parameter $f$. For $n=1$ the local Rossby number is less than unity out to $50 \mathrm{~km}$ radius and exceeds unity only in localized regions at larger radii (not shown). The balanced model is used with the general understanding that the balanced nature of the flow may extend somewhat beyond the formal validity of the model. Further, we do not attempt to use the model in a quantitative, predictive sense, but rather to gain a basic, qualitative understanding of the nature of the asymmetries.

The relation of the vorticity and pressure perturbations further supports the balanced nature of wave number 1 asymmetries. Inspection of the quasi-stationary wave number 1 pressure and vorticity asymmetries indicates that these perturbations are connected by an elliptic inversion operator characteristic of a balanced flow (not shown). Inverting the vorticity (PV) field to recover the pressure distribution would be more stringent evidence of the balanced nature, but this is reserved for future work.

\subsubsection{Interpretation of the outer vortex tilt as a standing VRW pattern}

The evidence presented above strongly suggests that the tilt evolution of the TC in our warm-rain experiment in the first $18-24 \mathrm{~h}$ is governed to zero order by a quasi-stationary, slowly decaying wave number 1 VRW pattern. Figure 15a illustrates the horizontal structure of the vorticity pattern at low levels above the BL. A strong dipole with an approximate $\mathrm{N}-\mathrm{S}$ orientation is found in the inner core, representing the inner vortex tilt. A weaker dipole is found outside of the eyewall. This weaker, but more extensive dipole is associated with the tilt of the outer vortex ${ }^{10}$. It has been noted earlier by Jones (1995, her Fig. 6a, here reproduced as Fig. 16b) and Reasor et al. (2004, their Fig. 10) that the tilt of the outer part of the vortex can be significantly larger than that of the inner core. In our experiment the larger tilt of the outer vortex is

\footnotetext{
${ }^{10}$ The reverse sign of the vorticity dipole in the inner core is associated with a displacement of the vortex centre in tilt direction.
}

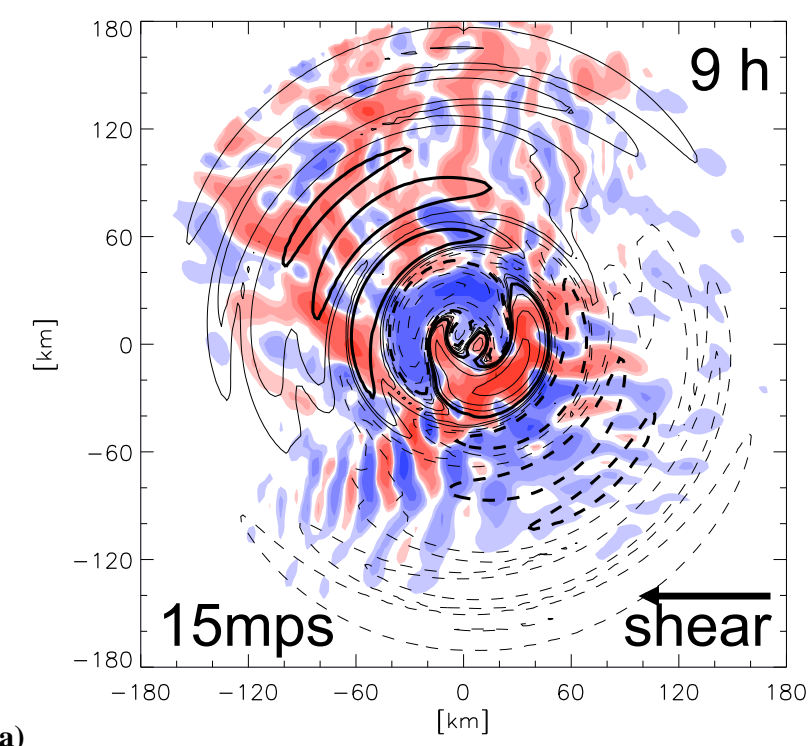

(a)

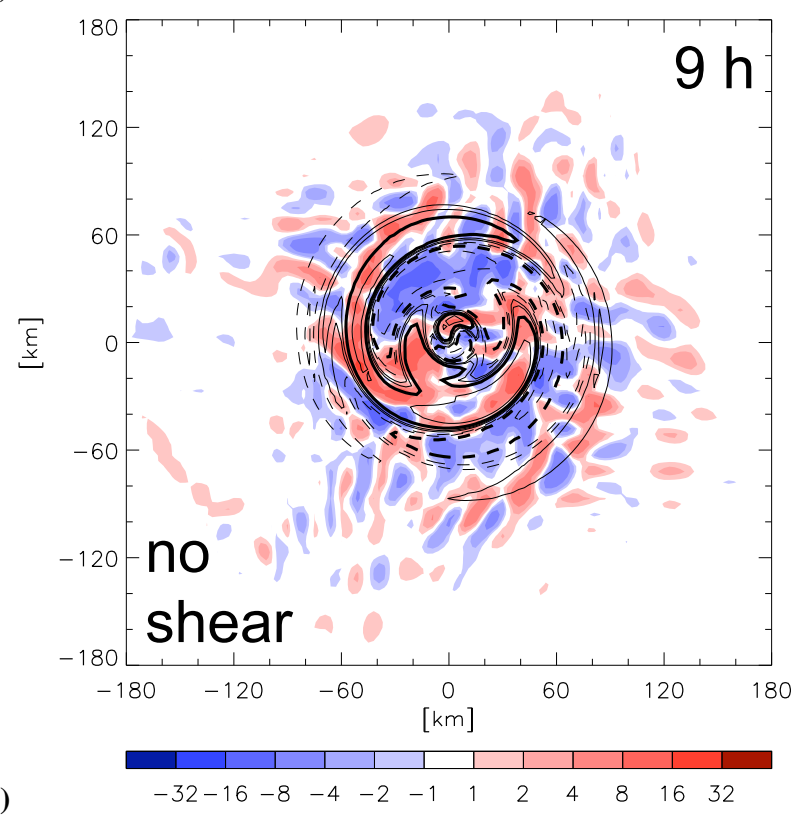

Fig. 15. Total vorticity (color, in $10^{-4} s^{-1}$ ) and wave number 1 asymmetry (contour, half the color scale, dashed for negative values, the thick contours denote $\pm 2 \times 10^{-4} \mathrm{~s}^{-1}$ ) above the BL at $2 \mathrm{~km}$ height at a representative time ( $9 \mathrm{~h}$ ) for the $15 \mathrm{mps}$ (a) and the no_shear (b) case. For the sheared case, most of the asymmetries reside inside the wave number 1 envelope out to large radii.

readily visible in the vertical vorticity cross-section along the tilt axis in Fig. 16a.

The wave number 1 vorticity pattern in the shear cases is in stark contrast to the pattern in the no_shear case. In the no_shear case, outside the eyewall, the wave number 1 vorticity pattern is dominated frequently by a trailing spiral (Fig. 15b). Inspecting subsequent time steps (not shown, but qualitatively similar to Fig. 12) the spiral is found to 


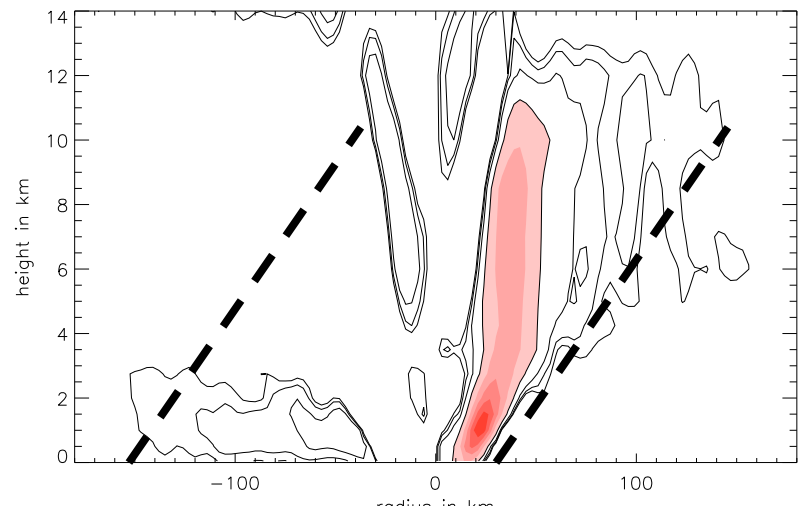

(a)

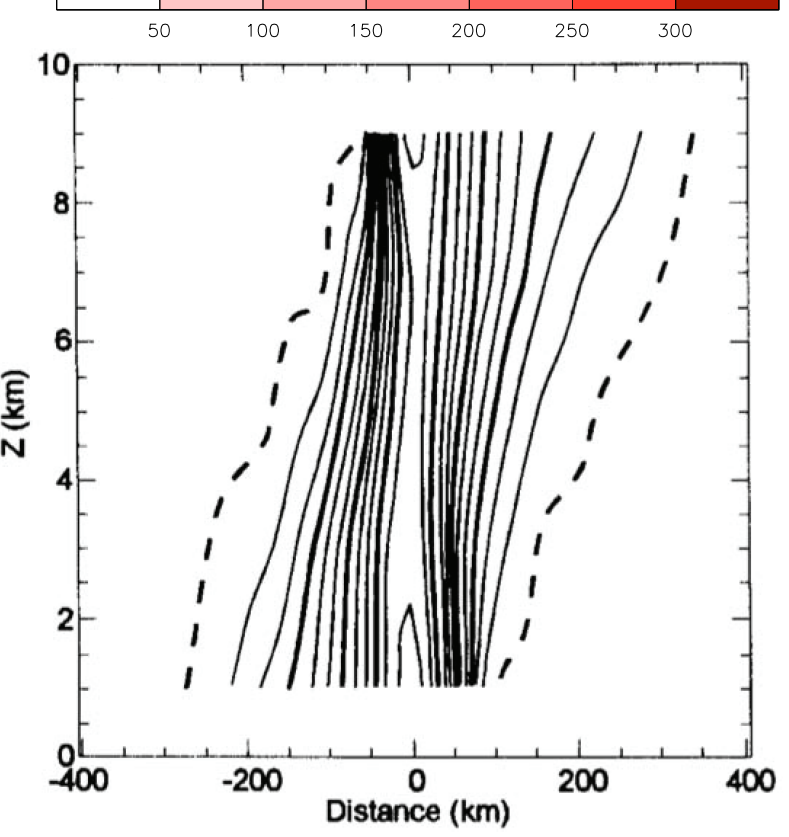

Fig. 16. (a) Vertical cross-section of positive, azimuthal wave number 1 vorticity asymmetry along the tilt axis looking approx. upshear ( $\approx$ North (left) to South (right)), averaged from $2 \mathrm{~h}$ to $7 \mathrm{~h}$. The color shaded region depicts the large asymmetry associated with the tilt of the inner core (color scale is in $10^{-5} \mathrm{~s}^{-1}$ ). Contours depict smaller values associated with the tilt of the outer vortex and are drawn at 5, 10, 20, and $50 \times 10^{-5} \mathrm{~s}^{-1}$. (b) Fig. 6a from Jones (1995): a vertical cross section of vorticity along the tilt axis of a vortex in a dry primitive Eq. numerical experiment. The inner core is virtually aligned while the outer core region exhibits a significant tilt (note the different aspect ratio).

propagate around the centre. Outside of the spiral, the vorticity anomalies are obviously not linked to a wave number 1 pattern. We regard the distinct differences between the wave number 1 characteristics in the no_shear and shear cases as further evidence that the wave number 1 asymmetry outside the eyewall in the shear cases is associated with the tilt of the outer vortex.

\subsection{Distribution of vertical motion outside of the eyewall}

In this subsection we will present evidence that the convective asymmetry outside the eyewall is forced by the tilt of the outer TC-vortex. We will show that the downdrafts that flux low $\theta_{e}$ air into the BL are intimately connected to this convective asymmetry. The distribution of the downward flux of low $\theta_{e}$ on the vortex-scale is thus governed to zero order by balanced TC dynamics.

\subsubsection{Ekman pumping by the outer-vortex-tilt vorticity anomaly}

The vorticity rich region associated with the outer vortex tilt provides a favorable environment for the formation of updrafts outside of the eyewall. Vorticity anomalies within the BL produce frictional convergence and thus force upward motion. The vertical motion at the top of the BL due to this mechanism, $w_{\text {Ekman }}$, can be estimated from simple Ekman pumping theory:

$w_{\mathrm{Ekman}}=\frac{1}{2} H_{\mathrm{BL}} \zeta^{\prime}$,

where $\zeta^{\prime}$ denotes the vertical vorticity wave number 1 asymmetry at the top of the BL. $\mathrm{H}_{\mathrm{BL}}$ denotes the height of the BL, set to $1.5 \mathrm{~km}$. Chen and Yau (2001) used the Ekman pumping argument to show the coupling of propagating near-core VRWs with vertical motion. The wave number 1 perturbation of vertical motion and $w_{\text {Ekman }}$ are compared at a representative time in Fig. 17. It is evident that the structure and the order of magnitude of the asymmetric vertical motion field can be explained to zero order by the simple Ekman pumping argument. On a more detailed level, however, the vertical motion asymmetry is locally up to a factor of 4 stronger than $w_{\text {Ekman }}$. This discrepancy can be traced to the balanced boundary layer approximation used to derive the Ekman relation (see, e.g., Smith and Montgomery (2008)). The wave number 1 envelope of vertical motion - containing the convective asymmetry outside of the eyewall - can therefore be considered to be forced by the quasi-steady vorticity asymmetry associated with the tilt of the outer vortex.

\subsubsection{Swirling of the updrafts and downdraft formation}

The general vertical structure of the vertical motion outside of the eyewall (at $75 \mathrm{~km}$ radius) is illustrated in Fig. 18a. The updrafts are predominantly rooted in the BL downshear to downshear-right (between $250^{\circ}$ and $330^{\circ}$ ). Due to advection by the swirling winds, ascent is along a slanted path ${ }^{11}$ The slope of $\theta_{e}$ isentropes - in particular the $360 \mathrm{~K}$ contour -

\footnotetext{
${ }^{11}$ The aspect ratio of the horizontal and vertical length scales is $\approx 20$. Assuming an azimuthal wind speed of $50 \mathrm{~ms}^{-1}$ this yields an average vertical wind speed of $2.5 \mathrm{~ms}^{-1}$, consistent with the values found in Fig. 18a.
} 


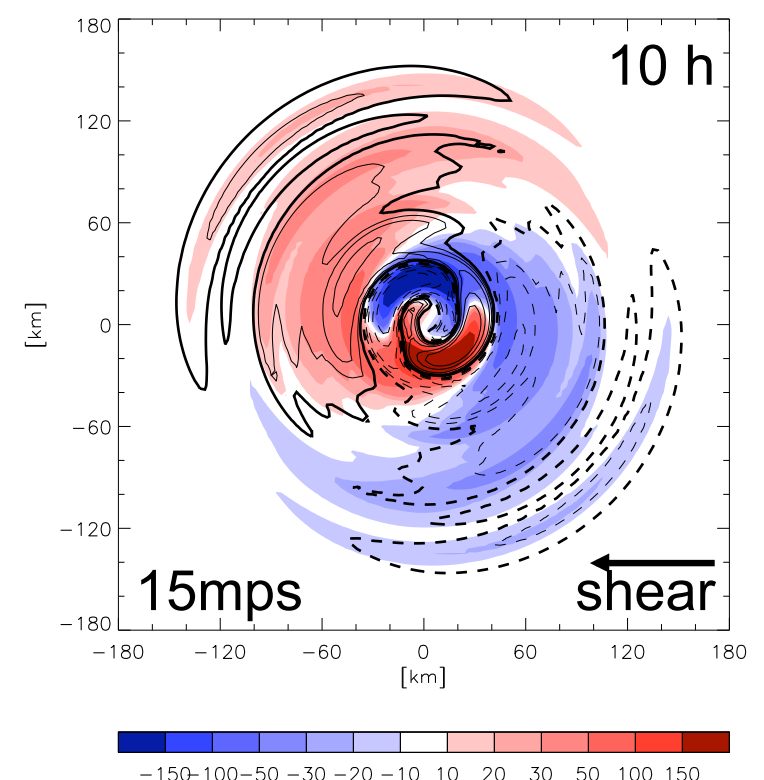

Fig. 17. Wave number 1 asymmetry of vertical motion at $1.5 \mathrm{~km}$ (color, in $\mathrm{cm} \mathrm{s}^{-1}$ ) and corresponding $w_{\text {Ekman }}$ (see text for definition, contours) at a representative time $(10 \mathrm{~h})$. Contours are drawn at half the scale of the color bar, negative values are dashed. The $\pm 5 \mathrm{cms}^{-1}$ contour is highlighted.

indicates that air parcels rise almost pseudo-adiabatically. An extended region of downdrafts is found underneath and downwind of the updrafts, in the downshear-left to upshear quadrant of the storm. The downdrafts most likely form by evaporation of rain falling out of the sloping updrafts into the unsaturated air below, possibly enhanced by water loading.

A pool of very low $\theta_{e}$ air is found between $140^{\circ}$ and $180^{\circ}$ azimuth below $4 \mathrm{~km}$, extending into the BL. There is an indication that this pool is connected to the mid-level $\theta_{e}$ minimum between $5 \mathrm{~km}$ and $8 \mathrm{~km}$, consistent with the tenet that the low $\theta_{e}$ air originates from the mid-levels. Low-level $\theta_{e}$ values are frequently found to be smaller than the values of the mid-level minimum, in particular in the $20 \mathrm{mps}$ case (not shown). Non-pseudo-adiabatic processes, e.g. precipitative flux (Tripoli and Cotton, 1981), may therefore play a role also. A strong azimuthal $\theta_{e}$ gradient is found between the region of updraft generation and the area where downdrafts reach the BL. At $1 \mathrm{~km}$ height the difference between the two regions at the time presented is $17 \mathrm{~K}$.

The pattern of up- and downdrafts can be considerably more complex at some individual times. Regardless of fluctuations in the detailed structure, the general structure is found to be persistent with time. This notion is supported by plotting the time average of the fields: all the features discussed above are still present in a $6 \mathrm{~h}$ average (Fig. 18b). This quasi-steadiness of the up- and downdraft pattern is an important difference to the no_shear case. Up- and downdraft couplets associated with wave number 1 VRWs are found in

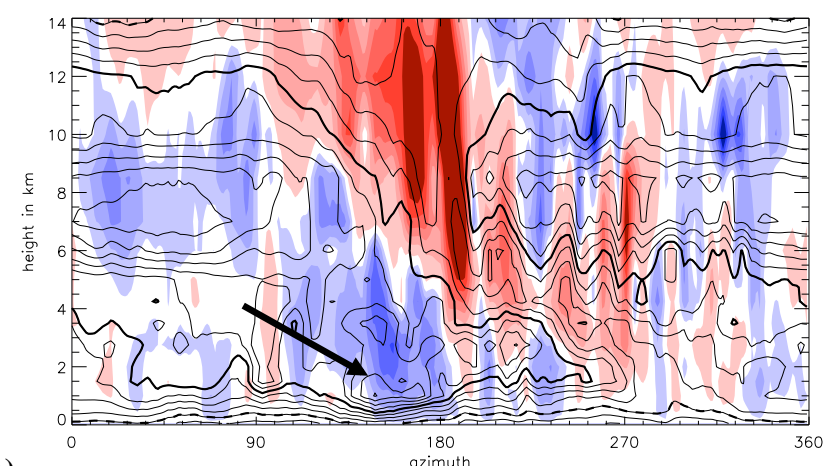

(a)

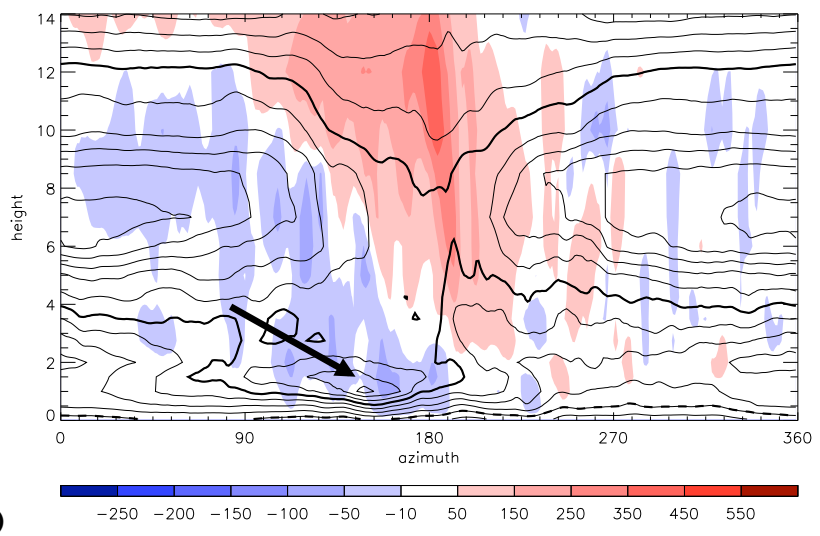

Fig. 18. Azimuth-height cross-section of vertical motion (color, in $\mathrm{cms}^{-1}$ ) and $\theta_{e}$ (contours, every $2.5 \mathrm{~K}, 350 \mathrm{~K}$ thick, $360 \mathrm{~K}$ dashed) along a radius of $75 \mathrm{~km}$ for the $15 \mathrm{mps}$ case. Looking South from the storm centre, $90^{\circ}$ is East and $270^{\circ}$ is West. A snapshot at time $9 \mathrm{~h}$ is shown in (a), a 6 h-average $(7 \mathrm{~h}-12 \mathrm{~h})$ is depicted in (b). The arrow highlights low $\theta_{e}$ values associated with the downdraft pattern.

the no_shear case also (Fig. 19a); these "rain bands" propagate azimuthally and the associated downward flux of low $\theta_{e}$ is far less pronounced. As might be expected, in the time averaged sense the azimuthal structure is virtually homogeneous for the no_shear case (Fig. 19b). The quasi-steadiness of the convective pattern in the sheared cases indicates that the convection forms in response to a quasi-steady forcing, consistent with our hypothesis that the tilt of the outer vortex provides this forcing.

We speculate that the persistent location and the banded structure (cf. Fig. 11) of the updraft-downdraft couplet promote the pronounced $\mathrm{BL} \theta_{e}$ depression in a Lagrangian sense. The basis for this speculation is that $\mathrm{BL}$ air parcels that spiral inward within the downdraft region experience a continuous frustration of the entropy uptake from the ocean surface. We further note that the persistent, strong, vertical gradient of $\theta_{e}$ at the top of the BL makes the intrusion of low $\theta_{e}$ air into the BL by downdrafts particularly effective. The existence of a pool of low $\theta_{e}$ air just above the BL implies also that air parcels do not need to descend from the midlevel minimum into the BL in a single downdraft plume to yield the observed $\theta_{e}$ depression. 


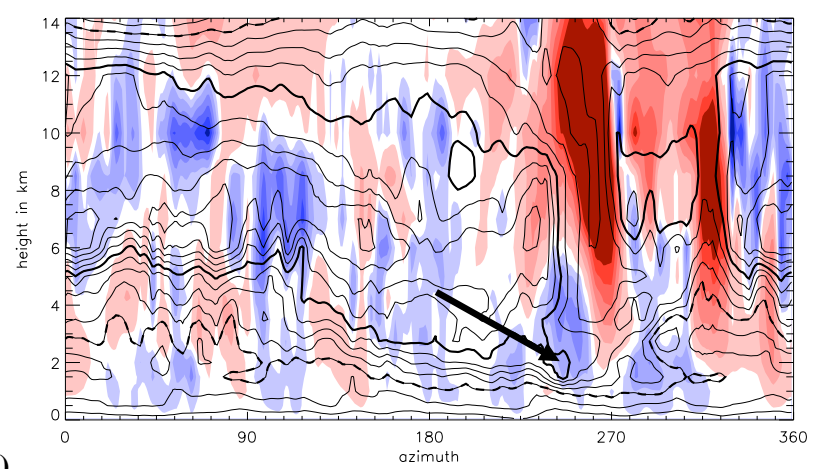

(a)

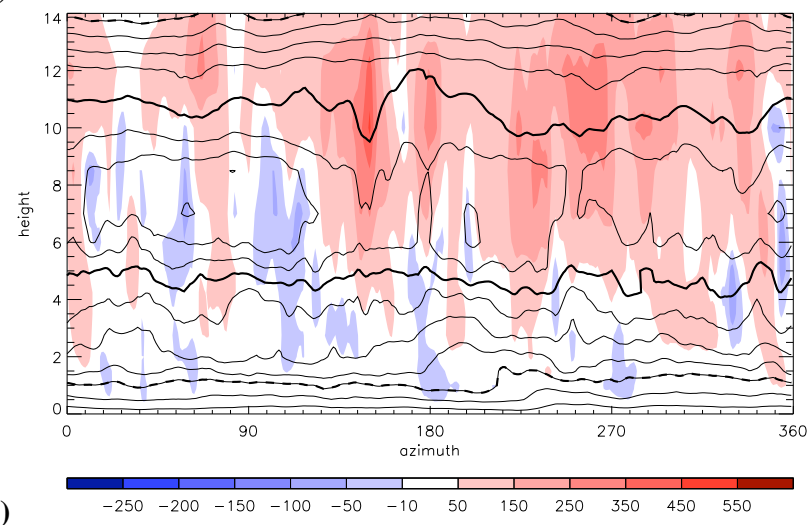

Fig. 19. Same as Fig. 18, but for the no_shear case.

\section{An interpretation of storm recovery and shear evolution}

In our experiments all storms re-intensify after the initial decrease in intensity. A comparison of the intensity and tilt magnitude time series show that the re-intensification in general coincides with the realignment of the TC. We argue that the reduction of the vortex tilt reduces the forcing of the convective asymmetry outside of the eyewall and thus hinders the formation of the quasi-stationary downdraft pattern. As the energy intake from the ocean surface is no longer considerably frustrated, the storm is able to re-intensify. The temporal evolution of the vertical wind shear somewhat complicates the interpretation of the recovery process. In a control experiment without a TC the vertical wind shear in thermal wind balance remains constant over the whole integration period. Due to the interaction with the TC, however, the shear magnitude and direction can be significantly modified (e.g. Wong and Chan, 2004). In the following we focus on the evolution of the shear magnitude that is representative of the near-core region.

Vertical wind shear is calculated as the vector difference between the average horizontal winds on a disc around the storm centre at $12 \mathrm{~km}$ and $1.5 \mathrm{~km}$ height, an approximation to the commonly used $200 \mathrm{hPa}$ and $850 \mathrm{hPa}$ levels. A disc with $120 \mathrm{~km}$ radius is used as this is the largest radius for which data from the innermost domain is available for all cases at all times ${ }^{12}$.

First it is noted that a northerly shear component develops in the shear cases (not shown), consistent with the southward motion component (Fig. 6). This northerly shear can be attributed to the downshear displacement of the outflow anomaly (e.g. Wu and Emanuel, 1993).

The shear magnitude in the near-core region can reach 5$7 \mathrm{~ms}^{-1}$ even without environmental shear (Fig. 20). This illustrates that internal storm dynamics can project considerably onto this shear metric. In the $20 \mathrm{mps}$ case the shear magnitude decreases considerably after the first $12 \mathrm{~h}$. Subsequently, shear values range between 6 and $11 \mathrm{~ms}^{-1}$. The pattern of shear evolution is similar in the $15 \mathrm{mps}$ case, with slightly smaller values than in the $20 \mathrm{mps}$ case. Shear values in the $10 \mathrm{mps}$ case range between $3 \mathrm{~ms}^{-1}$ and $11 \mathrm{~ms}^{-1}$ throughout the experiment with a peak of $16 \mathrm{~ms}^{-1}$ around $16 \mathrm{~h}$.

The left-of-shear tilt equilibrium is considered an optimal configuration because the shear imposed by the vortex tilt opposes the environmental shear (e.g. Jones, 1995; Reasor et al., 2004). In our experiment the vortex settles in the leftof-shear equilibrium within the first $1-2 \mathrm{~h}$ after the shear is imposed, well before the decrease of shear magnitude. We do not find a considerable shear reduction in the first few hours of our experiment. It is possible that using a radial range of $120 \mathrm{~km}$ for the shear calculation underestimates the shear reduction in the inner core. It is obvious, however, that another mechanism is involved in the shear reduction in our $20 \mathrm{mps}$ and $15 \mathrm{mps}$ cases also.

We hypothesise that an intense TC is able to axisymmetrise the storm relative flow in the near-core environment to some degree. The shear that affects the core of the system is thus significantly reduced. It stands to reason that the storm can more completely re-align and thus reintensify in the reduced-shear environment. It should be kept in mind that the shear flow in our experiment is only forced by the boundary conditions of the outer domain. We speculate that the ability of the vortex to reduce the near-core shear is less pronounced when the shear is associated with a dynamically strong weather system, e.g. an approaching upper-level trough.

This brief discussion suggests that a strong TC is affected by much less shear than what is imposed on the synoptic scale. We hypothesise that it is the shear within the Lagrangian boundary of the storm that controls the inner core dynamics. The important question of what determines the region in which the TC actually "feels" the vertical shear will be addressed in upcoming work.

\footnotetext{
${ }^{12} \mathrm{The} \mathrm{TC}$ in the $20 \mathrm{mps}$ case moves rather close to the boundary of the innermost domain at the end of the experiment.
} 


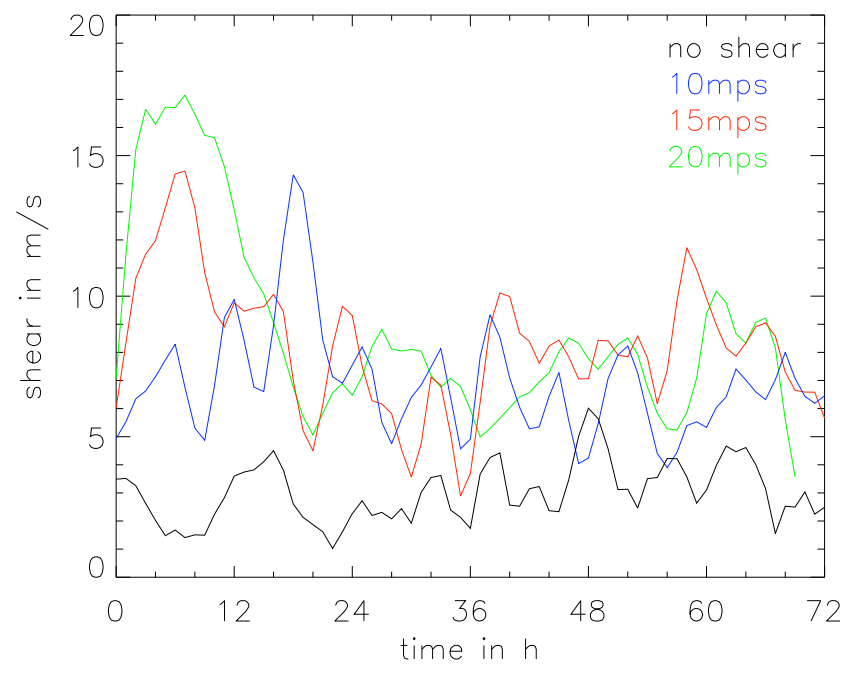

Fig. 20. Magnitude of the near-core shear for the no_shear (black), $10 \mathrm{mps}$ (blue), $15 \mathrm{mps}$ (red), and 20mps (green) case. Shear is calculated as the vector difference between the horizontal wind at $12 \mathrm{~km}$ and $1.5 \mathrm{~km}$ height, averaged horizontally over a disc around the storm centre with a radius of $120 \mathrm{~m}$. For clarity, the $3 \mathrm{~h}$ running mean of the time series is shown.

\section{Observational support and further discussion on convective asymmetries}

Before the final conclusions are presented, we support our new paradigm with observations of vertically sheared TCs in the real atmosphere. Some more discussion on the formation of the convective asymmetry outside of the eyewall is provided also.

\subsection{Asymmetric convection and associated downdraft patterns in the real atmosphere}

The convective asymmetry outside of the eyewall in our experiment is reminiscent of the stationary band complex (SBC) defined by Willoughby et al. (1984). These authors consider the relationship between the SBC and the low-level storm relative flow, not explicitly the relationship with vertical shear. They note, however, that low-level storm relative flow requires a difference between the environmental flow at the steering level, usually found at mid-levels, and the lowlevel flow, and thus vertical shear. The orientation of the convective asymmetry with respect to the low-level storm relative flow in our experiment is consistent with the results of Willoughby et al. (1984), although care must be taken when comparing their radar reflectivity data with our model vertical motion. The observations provided in Willoughby et al. (1984) thus support our notion of asymmetric convective activity outside of the eyewall in sheared TCs. Ample further evidence of the formation of this convective asymmetry in the satellite representation of real TCs in vertical shear can

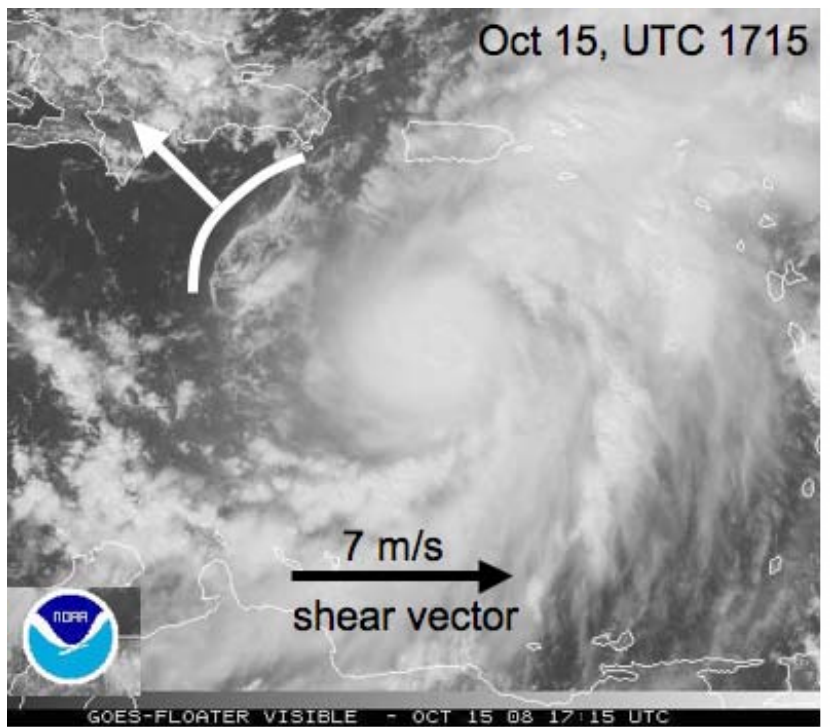

Fig. 21. Visible satellite imagery of Hurricane Omar south of Puerto Rico on 15 Oct 2008 at 17:15 UTC (courtesy of NOAA). The shear direction is from the West. Shear direction and magnitude are estimated from Chris Velden's email shear product at CIMMS (Velden, personal communication, 2009). The arc cloud band to the Northwest of the storm is highlighted by the white curve. The white arrow indicates the movement of the band relative to the storm centre.

be found in the literature, e.g. in Black et al. (2002, Hurricane Olivia (1994), their Fig. 14).

The formation of downdrafts and the associated thermodynamic impact on the BL in vertically sheared TCs has not yet been a focus of observational studies. Powell (1990, among others) has documented downdrafts and the associated modification of the $\operatorname{BL} \theta_{e}$ distribution on the scale of individual rain bands. A widespread flooding of the BL with low $\theta_{e}$ air by persistent downdrafts has not been observed yet with in situ data. Here we provide two observations that indicate the existence of persistent downdrafts in the downshear-left to upshear quadrant of real storms. In Hurricane Omar (2008) an arc cloud band propagating away from the storm centre in the downshear-left to upshear quadrant is found in visible satellite imagery (Fig. 21). Arc cloud bands indicate persistent downdrafts. Halverson et al. (2006), although focusing on the upper-level warm core structure of Hurricane Erin (2001) in vertical shear, present a map of surface divergence (their Fig. 4, here reproduced as Fig. 22). A region of strong surface divergence is evident from the contiguous purple arc-like feature in Fig. 22, with an estimated length and width of $\mathcal{O}(400 \mathrm{~km}) \times \mathcal{O}(50 \mathrm{~km})$. Halverson et al. did not comment on this vortex-scale feature. The feature is found downwind of a region of convergence that supports a spirallike convective asymmetry dominated by wave number 1 . It was this latter convective asymmetry that was emphasised in Halverson et al. (2006). Divergence at the surface implies, 


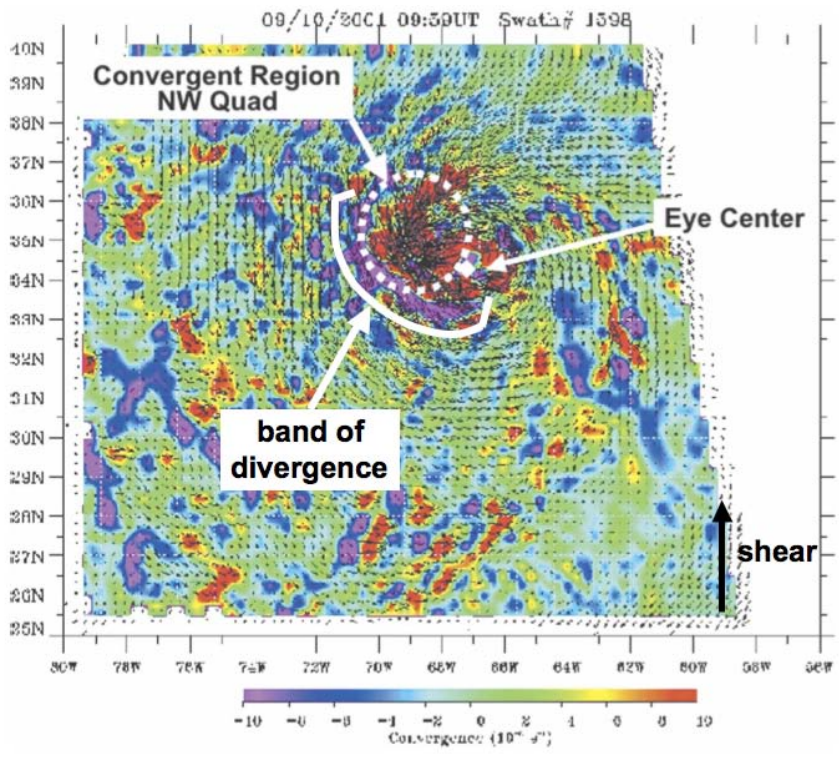

Fig. 22. Figure 4 from Halverson et al. (2006): Convergence at sea level (color) and storm relative motion (arrows) for Hurricane Erin (2001) at 10:00 UTC 10 September, obtained from gridded QuickSCAT data. Red areas denote convergence, and purple shows divergence. The authors of the present study have highlighted the band of divergence and indicated the approximate shear direction at this time. See comment in Halverson et al. (2006) about potential impact of rain contamination.

by mass continuity, downward motion above. At this time the direction of the shear is approximately from the South. The orientation of the convective asymmetry and the adjacent band of downdrafts is as predicted by our new paradigm.

\subsection{Formation of the convective asymmetry outside the eyewall}

Willoughby et al. (1984) provide an appealing explanation for the formation of the convective asymmetry outside of the eyewall of a mature TC based on the idea of a Lagrangian boundary between the swirling winds of the vortex and the low-level storm relative flow. To the best of our knowledge, however, no attempt has been reported in the literature to support Willoughby et al.'s ideas with higher resolution data. In the present study, we provide a complementary explanation based on the balanced dynamics of an intense vortex immersed in a vertically sheared flow: The convective asymmetry is forced by the vorticity asymmetry associated with the tilt of the outer vortex. In the context of simplified boundary layer models, asymmetric frictional forcing due to storm motion has been shown to also force convective asymmetries (Shapiro, 1983; Kepert, 2001; Kepert and Wang, 2001). In these studies the asymmetries due to storm motion appear to be confined to the inner core. Unpublished results by Ooyama (1986), however, indicate that frictionally forced asymmetries in the convergence pattern can extend outward to a radius of $\mathcal{O}(100 \mathrm{~km})$. It seems therefore possible that asymmetric frictional forcing further modulates the convective asymmetry outside of the eyewall.

In all of these studies on asymmetric frictional forcing, the assumptions are made that the pressure distribution at the top of the BL is symmetric and that the developing asymmetries can be found as a stationary solution. It is not clear to us if these assumptions hold for a sheared, and thus inherently asymmetric TC undergoing pronounced intensity modification. An observational study by Corbosiero and Molinari (2003) does not support the idea that storm motion plays an important role for the structure of a TC in vertical shear. Using lightning data in 303 sample time periods of $35 \mathrm{TCs}$, Corbosiero and Molinari concluded that the distribution of asymmetric convection is governed by the direction of environmental shear rather than the motion vector, at least for moderate to strong shear values.

Clearly, more research is necessary to disentangle the individual contributions to the formation of what has been dubbed the "stationary band complex".

\section{Conclusions}

An important roadblock to improved intensity forecasts is our incomplete understanding of the interaction of a TC with the environmental flow. In this study we have thus revisited the canonical problem of vertically sheared TCs in idealised numerical experiments. A TC that was spun up in a quiescent environment is subjected to a unidirectional shear profile with cosine vertical structure. We considered moderate to strong shear values and an intense tropical cyclone. On the basis of the results presented herein we speculate that our results hold qualitatively for less intense storms in weaker shear also. In all experiments the $\mathrm{TC}$ is resilient but significant differences in the intensity evolution occur.

The evidence presented in this article points to an unsung pathway to intensity modification by vertical shear: Vertical shear impacts the energy cycle of a TC at its most vulnerable part - the inflow layer of the storm. Flushing of low $\theta_{e}$ air into the BL outside of the eyewall significantly depresses BL $\theta_{e}$ values. The BL $\theta_{e}$ values do not recover sufficiently before reaching the eyewall, leading to lower $\theta_{e}$ values within the eyewall convection. This implies a frustration of the thermodynamic cycle of the TC heat engine. Less kinetic energy can be generated and a decrease in storm intensity is expected. We show evidence that the thermodynamic impact on the BL likely dominates over possible adverse processes operating at mid- to upper-levels. The close association between the flushing of the BL with low $\theta_{e}$ air and the intensity evolution of the storm further supports our hypothesis.

The downdrafts that flush the BL with low $\theta_{e}$ air are tied to a quasi-stationary, azimuthal wave number 1 convective asymmetry outside of the eyewall. This convective 
asymmetry and the associated downdraft pattern extends outwards to approx. $150 \mathrm{~km}$. Downdrafts occur on the vortex scale and form where rain falls out of the helical updrafts into the unsaturated air below. The formation location indicates that evaporative cooling, possibly enhanced by water loading, is the dominant downdraft formation mechanism. We argue that the quasi-steadiness of the updraft-downdraft pattern has important ramifications for the downward flux of low $\theta_{e}$ air into the BL: Underneath the persistent downdrafts a reservoir of low $\theta_{e}$ air builds up at the top of the BL. Downdrafts that tap this reservoir are particularly effective in flushing low $\theta_{e}$ air into the BL.

Our results point to an important connection between the convective asymmetry outside of the eyewall - and thus the thermodynamic impact in the near-core boundary layer - and the asymmetric balanced dynamics governing the evolution of the TC vortex subject to vertical shear. It is argued that the tilt of the sheared TC in our warm-rain experiment is represented to zero order by a standing VRW pattern, extending outwards to radii of $150 \mathrm{~km}-200 \mathrm{~km}$. Previous studies have shown that propagating rain bands in the near-core region of an unforced model TC can be approximately described as balanced features (Chen and Yau, 2001; Wang, 2002; Chen et al., 2003). Here we show in a warm-rain numerical experiment that in a shear-forced TC the prevalence of balanced motion can extend outwards to considerably larger radii. Work is in progress to further examine the balanced nature of the vortex dynamics in that region.

The tilt of the outer vortex in our experiments is considerably larger than the tilt of the eyewall, consistent with previous numerical experiments of dry vortices (Jones, 1995; Reasor et al., 2004). This outer-vortex tilt constitutes a wave number 1 vorticity asymmetry near the top of the BL outside of the eyewall out to radii of $150 \mathrm{~km}-200 \mathrm{~km}$. Vorticity anomalies within the BL produce frictional convergence and thus force upward motion. The positive vorticity anomaly associated with the standing wave number 1 pattern thus constitutes a preferred region of updraft formation. Consistent with results of Chen and Yau (2001), a simple Ekman pumping argument provides a zero order explanation for the associated wave number 1 vertical motion asymmetry. Convective feedback amplifies the updrafts and favors the smaller scales. Within the wave number 1 envelope the convection takes the form of bands and clusters.

In a modeling study like this, the formation of downdrafts might be sensitive to the background sounding and the microphysics parameterisation. Both sensitivities should be investigated in future numerical experiments. Using a more complete microphysics scheme - in particular including the ice phase - downdraft formation may be modified, inter alia, by cooling associated with melting and by the fallout of frozen hydrometeors with terminal velocities different from rain. Significant downdrafts, however, appear to form in our experiment below the freezing level at 5-6 km (Fig. 18a). In conjunction with the observational support given in Sect. 7.1, we thus believe that the downdraft formation and the associated significant $\mathrm{BL} \theta_{e}$ depression is not an artifact of the warm rain scheme.

We speculate that a potential sensitivity to the initial sounding has a physical meaning and might have important implications for intensity forecasts. The flushing of the BL with low $\theta_{e}$ air might be particularly pronounced when the asymmetric convection outside the eyewall interacts with anomalously dry low- to mid-level air in the downshear-left semicircle. Observations of the thermodynamic properties of the environmental air in this region should prove helpful towards obtaining a more complete understanding of intensity change associated with vertical shear.

Acknowledgements. We appreciate collaboration with Kerry Emanuel (MIT) and insightful discussions with Jack Beven (NHC). This research was performed while the first author held a National Research Council Research Associateship Award at the Naval Postgraduate School. This work is supported by NFS grants ATM0649946 and ATM-0715426, the ONR grant N001408WR20129 and by the US Naval Postgraduate School in Monterey, California. The comments from John Molinari, one anonymous reviewer, and Brian Tang have helped improve the presentation of our results.

Edited by: W. E. Asher

\section{References}

Bender, M. A.: The effect of relative flow on the asymmetric structure in the interior of hurricanes, J. Atmos. Sci., 54, 703-724, 1997.

Black, M. L., Gamache, J. F., Marks, F. D., Samsury, C. E., and Willoughby, H. E.: Eastern Pacific Hurricanes Jimena of 1991 and Olivia of 1994: The effect of vertical shear on structure and intensity, Mon. Wea. Rev., 130, 2291-2312, 2002.

Black, P. G., D’ Asaro, E. A., Drennan, W. M., French, J. R., Niiler, P. P., Sanford, T. B., Terrill, E. J., Walsh, E. J., and Zhang, J. A.: Air-sea exchange in hurricanes: Synthesis of observations from the Coupled Boundary Layer Air-Sea Transfer experiment, Bull. Am. Meteorol. Soc., 88, 357-374, 2007.

Braun, S. A. and Wu, L.: A numerical study of Hurricane Erin (2001). Part II: Shear and the organization of eyewall vertical motion, Mon. Wea. Rev., 135, 1179-1194, 2007.

Braun, S. A., Montgomery, M. T., and Pu, Z.: High-resolution simulation of Hurricane Bonnie (1998). Part I: The organization of eyewall vertical motion, J. Atmos. Sci., 63, 19-42, 2006.

Carr, L. E. and Williams, R. T.: Barotropic vortex stability to perturbations from axisymmetry, J. Atmos. Sci., 46, 3177-3191, 1989.

Chen, Y. and Yau, M. K.: Spiral bands in a simulated hurricane. Part I: Vortex Rossby wave verification, J. Atmos. Sci., 58, 21282145, 2001.

Chen, Y., Brunet, G., and Yau, M. K.: Spiral Bands in a Simulated Hurricane. Part II: Wave Activity Diagnostics, J. Atmos. Sci., 60, 1239-1256, 2003.

Clark, T. L. and Farley, R. D.: Severe downslope windstorm calculations in two and three spatial dimensions using anelastic interactive grid nesting: A possible mechanism for gustiness, J. Atmos. Sci., 41, 329-350, 1984. 
Corbosiero, K. L. and Molinari, J.: The relationship between storm motion, vertical wind shear, and convective asymmetries in tropical cyclones, J. Atmos. Sci., 60, 366-376, 2003.

Cotton, W. R. and Coauthors: RAMS 2001: Current status and future directions, Meteor. Atmos. Phys., 82, 5-29, 2003.

Cram, T. A., Persing, J., Montgomery, M. T., and Braun, S. A.: A Lagrangian trajectory view on transport and mixing processes between the eye, eyewall, and environment using a highresolution simulation of Hurricane Bonnie (1998), J. Atmos. Sci., 64, 1835-1856, 2007.

Davis, C. A., Jones, S. C., and Riemer, M.: Hurricane vortex dynamics during Atlantic extratropical transition, J. Atmos. Sci., 65, 714-736, 2008.

DeMaria, M.: The effect of vertical shear on tropical cyclone intensity change, J. Atmos. Sci., 53, 2076-2088, 1996.

Emanuel, K. A.: An air-sea interaction theory for tropical cyclones. Part I: Steady-state maintenance, J. Atmos. Sci., 43, 585-605, 1986.

Emanuel, K. A.: The theory of hurricanes, Annu. Rev. Fluid Mech., 23, 179-196, 1991.

Frank, W. M. and Ritchie, E. A.: Effects of vertical wind shear on the intensity and structure of numerically simulated hurricanes, Mon. Wea. Rev., 129, 2249-2269, 2001.

Halverson, J. B., Simpson, J., Heymsfield, G., Pierce, H., Hock, T., and Ritchie, L.: Warm core structure of Hurricane Erin diagnosed from high altitude dropsondes during CAMEX-4, J. Atmos. Sci., 63, 309-324, 2006.

Hill, G. E.: Factors controlling the size and spacing of cumulus clouds as revealed by numerical experiments, J. Atmos. Sci., 31, 646-673, 1974.

Jones, S. C.: The evolution of vortices in vertical shear. I: Initially barotropic vortices, Q. J. R. Meteorol. Soc., 121, 821-851, 1995.

Jones, S. C.: The evolution of vortices in vertical shear. III: Baroclinic vortices, Q. J. R. Meteorol. Soc., 126, 3161-3185, 2000.

Jordan, C. L.: Mean sounding for the West Indies area, J. Atmos. Sci., 15, 91-97, 1958.

Kepert, J.: The dynamics of boundary layer jets within the tropical cyclone core. Part I: Linear theory, J. Atmos. Sci., 58, 2469 2484, 2001.

Kepert, J. and Wang, Y.: The dynamics of boundary layer jets within the tropical cyclone core. Part II: Nonlinear enhancement, J. Atmos. Sci., 58, 2485-2501, 2001.

Kessler, E.: On the distribution and continuity of water substance in atmospheric circulations, Meteor. Mon. 32, Amer. Meteor. Soc., 1969.

Klemp, J. B. and Wilhelmson, R. B.: The simulation of threedimensional convective storm dynamics, J. Atmos. Sci., 35, 1070-1096, 1978.

Lilly, D. K.: On the numerical simulation of buoyant convection, Tellus, 14, 148-172, 1962.

Makarieva, A. M., Gorshkov, V. G., Li, B.-L., and Nobre, A. D.: A critique of some modern applications of the Carnot heat engine concept: the dissipative heat engine cannot exist, Proc. Roy. Soc. A, in print, 2010.

Melander, M. V., McWilliams, J. C., and Zabusky, N. J.: Axisymmetrization and vorticity-gradient intensification of an isolated two-dimensional vortex through filamentation, J. Fluid Mech., 178, 137-159, 1987.

Montgomery, M. T. and Kallenbach, R. J.: A theory for vortex
Rossby-waves and its application to spiral bands and intensity changes in hurricanes, Q. J. R. Meteorol. Soc., 123, 435-465, 1997.

Montgomery, M. T., Nguyen, V. S., Persing, J., and Smith, R. K.: Do tropical cyclones intensify by WISHE?, Q. J. R. Meteorol. Soc., 135, 1697-1714, 2009.

Nguyen, S. V., Smith, R. K., and Montgomery, M. T.: Tropicalcyclone intensification and predictability in three dimensions, Q. J. R. Meteorol. Soc., 134, 563-582, 2008.

Ooyama, K. V.: A spectral prediction model on nested domains and its application to asymmetric flow in the hurricane boundary layer, in: International Symposium on Short and Medium Range Numerical Weather Prediction, WMO/IUGG, Tokyo, Japan, 1986.

Pielke, R. A. and Coauthors: A comprehensive meteorological modeling system - RAMS, Meteor. Atmos. Phys., 49, 69-91, 1992.

Powell, M. D.: Boundary layer structure and dynamics in outer hurricane rainbands. Part II: Downdraft modification and mixed layer recovery, Mon. Wea. Rev., 118, 918-938, 1990.

Reasor, P. D. and Montgomery, M. T.: Three-dimensional alignment and corotation of weak, TC-like vortices via linear vortex Rossby waves, J. Atmos. Sci., 58, 2306-2330, 2001.

Reasor, P. D., Montgomery, M. T., and Grasso, L. D.: A new look at the problem of tropical cyclones in vertical shear flow: Vortex resiliency, J. Atmos. Sci., 61, 3-22, 2004.

Rogers, R., Chen, S., Tenerelli, J., and Willoughby, H.: A numerical study of the impact of vertical shear on the distribution of rainfall in Hurricane Bonnie (1998), Mon. Wea. Rev., 131, 1577-1599, 2003.

Schecter, D. A. and Montgomery, M. T.: On the symmetrization rate of an intense geophysical vortex, Dyn. Atmos. Oceans, 37 55-88, 2003.

Schecter, D. A. and Montgomery, M. T.: Waves in a cloudy vortex, J. Atmos. Sci., 64, 314-337, 2007.

Schubert, W. H., Montgomery, M. T., Taft, R. K., Guinn, T. A., Fulton, S. R., Kossin, J. P., and Edwards, J. P.: Polygonal eyewalls, asymmetric eye contraction, and potential vorticity mixing in hurricanes, J. Atmos. Sci., 56, 1197-1223, 1999.

Shapiro, L. J.: The asymmetric boundary layer flow under a translating hurricane, J. Atmos. Sci., 40, 1984-1998, 1983.

Shapiro, L. J. and Montgomery, M. T.: A three-dimensional balance theory for rapidly rotating vortices, J. Atmos. Sci., 50, 3322 3335, 1993.

Simpson, R. H. and Riehl, H.: Mid-tropospheric ventilation as a constraint on hurricane development and maintenance, in: Proc. Tech. Conf. on Hurricanes, pp. D4.1-D4.10, Amer. Meteor. Soc., Miami, FL, 1958.

Smagorinsky, J.: General circulation experiments with the primitive equations, Mon. Wea. Rev., 91, 99-164, 1963.

Smith, R. K., Ulrich, W., and Sneddon, G.: On the dynamics of hurricane-like vortices in vertical-shear flows, Q. J. R. Meteorol. Soc., 126, 2653-2670, 2000.

Smith, R. K. and Montgomery, M. T.: Balanced boundary layers used in hurricane models, Q. J. R. Meteorol. Soc., 134, 13851395, 2008.

Smith, R. K., Montgomery, M. T., and Vogl, S.: A critique of Emanuel's hurricane model and potential intensity theory, Q. J. R. Meteorol. Soc., 134, 551-561, 2008. 
Tripoli, G. J. and Cotton, W. R.: The use of ice-liquid water potential temperature as a thermodynamic variable in deep atmospheric models, Mon. Wea. Rev., 109, 1094-1102, 1981.

Vecchi, G. A. and Soden, B. J.: Increased tropical Atlantic wind shear in model projections of global warming, Geophys. Res. Lett., 34, 2007.

Wang, Y.: Vortex Rossby Waves in a Numerically Simulated Tropical Cyclone. Part I: Overall Structure, Potential Vorticity, and Kinetic Energy Budgets, J. Atmos. Sci., 59, 1213-1238, 2002.

Wang, Y., Montgomery, M., and Wang, B.: How much vertical shear can a well-developed tropical cyclone resist?, in: Preprints of the 26th Conference on Hurricanes and Tropical Meteorology, pp. 100-101, Amer. Meteor. Soc., Miami, FL, 2004.
Willoughby, H. E., Marks, F. D., and Feinberg, R. J.: Stationary and moving convective bands in hurricanes, J. Atmos. Sci., 41, 3189-3211, 1984.

Wong, M. L. M. and Chan, J. C. L.: Tropical cyclone intensity in vertical wind shear, J. Atmos. Sci., 61, 1859-1876, 2004.

Wu, C.-C. and Emanuel, K. A.: Interaction of a baroclinic vortex with background shear: Application to hurricane movement, J. Atmos. Sci., 50, 62-76, 1993.

Wu, L. and Braun, S. A.: Effects of environmentally induced asymmetries on hurricane intensity: A numerical study, J. Atmos. Sci., 61, 3065-3081, 2004.

Zhang, J. A., Black, P. G., French, J. R., and Drennan, W. M.: First direct measurements of enthalpy flux in the hurricane boundary layer: The CBLAST results, Geophys. Res. Lett., 35, 2008. 